\title{
Cell-intrinsic functions of the transcription factor Bhlhe40 in activated B cells and T follicular helper cells restrain the germinal center reaction and prevent lymphomagenesis
}

René Rauschmeier ${ }^{1,10}$, Annika Reinhardt ${ }^{2,3,10}$, Charlotte Gustafsson ${ }^{4}$, Vassilis Glaros ${ }^{2,3}$, Artem V. Artemov $^{5,6}$, Reshma Taneja ${ }^{7}$, Igor Adameyko ${ }^{5,8}$, Robert Månsson ${ }^{4,9}$, Meinrad Busslinger $^{1,11^{*}}$, and Taras Kreslavsky ${ }^{2,3,11^{*}}$

1 - Research Institute of Molecular Pathology (IMP), Vienna Biocenter (VBC), Vienna, Austria

2 - Department of Medicine, Division of Immunology and Allergy, Karolinska Institutet, Karolinska University Hospital, Stockholm, Sweden

3 - Center for Molecular Medicine, Karolinska Institutet, Stockholm, Sweden

4 - Center for Hematology and Regenerative Medicine, Department of Laboratory Medicine, Karolinska Institutet, Sweden

5 - Department of Neuroimmunology, Medical University of Vienna, Vienna, Austria

6 - Institute for Regenerative Medicine, Sechenov First Moscow State Medical University, Moscow, Russian Federation

7 - Department of Physiology, Healthy Longevity Translational Research Program, Yong Loo Lin School of Medicine, National University of Singapore, Singapore

8 - Department of Physiology and Pharmacology, Karolinska Institutet, Stockholm, Sweden

9 - Hematology Center, Karolinska University Hospital, Stockholm, Sweden

10 - these authors contributed equally to the study

11 - corresponding authors

* - to whom correspondence should be addressed:

Taras Kreslavsky

Phone: +46761 124813

E-mail: Taras.Kreslavskiy@ki.se

Meinrad Busslinger

Phone: +43 1797303150

E-mail: Meinrad.Busslinger@imp.ac.at 


\section{ABSTRACT}

The generation of high-affinity antibodies against pathogens and vaccines requires the germinal center (GC) reaction - a process that relies on a complex interplay between specialized effector subsets of B and CD4 T lymphocytes - GC B cells and T follicular helper $\left(T_{F H}\right)$ cells. Intriguingly, several key positive regulators of the GC reaction are common for both cell types. Here, we report that the transcription factor Bhlhe40 is a crucial cell-intrinsic negative regulator affecting both the B and T cell sides of the GC reaction. In activated CD4 T cells, Bhlhe40 was required to restrain proliferation thus limiting the number of $\mathrm{T}_{\mathrm{FH}}$ cells. In B cells, Bhlhe40 executed its function in the first days after immunization by selectively restricting the generation of the earliest GC B cells but not of early memory B cells or plasmablasts. Conditional Bhlhe40 inactivation confirmed cell-autonomous functions of Bhlhe40 in both GC B and $T_{F H}$ cells, while the GC phenotype was further enhanced upon loss of Bhlhe40 in both cell types. This negative regulation of the $\mathrm{GC}$ reaction by Bhlhe40 was of crucial importance, as Bhlhe40-deficient mice with progressing age succumbed to a B cell lymphoma characterized by accumulation of monoclonal GC B-like cells and polyclonal $\mathrm{T}_{\mathrm{FH}}$ cells in various tissues. 


\section{INTRODUCTION}

Generation of high-affinity antibodies in response to pathogens and vaccines depends on a complex interplay between specialized subsets of activated antigen-specific B and T lymphocytes - germinal center (GC) B cells and T follicular helper $\left(T_{F H}\right)$ cells - that takes place in the course of the GC reaction. Upon activation by cognate antigen, B cells undergo a burst of proliferation and differentiate into plasmablasts (PBs), non-GC-derived memory B cells (MBCs), or GC B cells ${ }^{1}$. The latter cells populate GCs - temporary microanatomical structures that form around follicular dendritic cell (FDC) clusters in B cell follicles of secondary lymphoid organs. The GC reaction is an iterative process that involves repetitive cycles of B cell proliferation, hypermutation of their antibody genes by activation-induced cytidine deaminase (AID), competition of the mutated B cell clones for antigen and subsequent presentation of the processed antigen to a specialized T helper cell subset $-\mathrm{T}_{\mathrm{FH}}$ cells (reviewed $\mathrm{in}^{2,3,4}$ ). T cell help provided by $\mathrm{T}_{\mathrm{FH}}$ cells in response to this antigen presentation is crucial for the survival and expansion of the $\mathrm{B}$ cell clones with higher affinity. B cells eventually egress from the $G C$ reaction to give rise to $M B C$ s and high affinity plasma cells (PCs). GCs can be divided histologically into a light zone (LZ) where B cells interact with FDCs that capture and display antigen and $\mathrm{T}_{\mathrm{FH}}$ cells, and a dark zone (DZ) where proliferation and hypermutation takes place. The GC reaction therefore involves continuous recirculation of $B$ cells between the $L Z$ and the DZ. A wave of expression of the transcription factor Myc that is transiently induced in the LZ proportionally to the magnitude of $\mathrm{T}$ cell help ${ }^{5}$ drives proliferation of GC B cells in the $\mathrm{DZ}{ }^{6,7}$, in part through induction of the Myc target gene Tfap4 encoding the transcription factor $\mathrm{AP} 4^{8}$. While the $\mathrm{GC}$ response is absolutely required for the generation of high-affinity antibodies, active mutagenesis in highly proliferative cells comes at a price, as dysregulation of the GC reaction can lead to lymphomagenesis. Indeed, a wide variety of B cell lymphomas including follicular lymphoma, GC B cell-like diffuse large B cell lymphoma, Burkitt's lymphoma, Hodgkin's lymphomas, as well as a number of more rare lymphoma types all originate from the $\mathrm{GC}$ reaction ${ }^{9}$. 
For reasons that are not well understood, many key regulators of the GC reaction have cellintrinsic functions in both GC B cells and $\mathrm{T}_{\mathrm{FH}}$ cells. For example, mice deficient for the transcription factor Bcl6 completely lack GC responses, as Bcl6 is required for the differentiation of both $G C B$ cells and $T_{F H}$ cells $^{10,11,12,13,14}$. Although the molecular programs regulated by Bcl6 in GC B cells and $T_{F H}$ cells are very different ${ }^{15}$, in both cell types it functions in part through the direct repression of Prdm1 that encodes for the transcription factor Blimp $1^{16}$. Blimp1 interferes with the GC B cell molecular program by promoting PC differentiation and shifting the balance of $T$ helper cell differentiation from $T_{F H}$ to $T_{H 1} 17,18,19$. The transcription factor Irf4 also promotes PC differentiation when expressed at high levels, however moderate amounts of Irf4 are required for upregulation of Bcl6 during the early phases of the GC response $^{20,21}$ as well as for $T_{F H}$ differentiation ${ }^{22}$. The GC formation is completely abrogated in absence of the transcription factor Batf ${ }^{23}$ that regulates expression of $\mathrm{Bcl} 6$ and $\mathrm{Maf}^{24}$ in $\mathrm{T}_{\mathrm{FH}}$ cells and is required for immunoglobulin class-switch recombination and cell expansion in GC B cells ${ }^{25} 24$. The transcription factor Foxo1 regulates DZ specification in GC B cells ${ }^{26,27}$ and is required for late-stage GC $T_{F H}$ cell differentiation while inhibiting earlier steps of $\mathrm{T}_{\mathrm{FH}}$ development ${ }^{28}$. The list of such common regulators is not restricted to transcription factors as mutations in Rc3h1 encoding the RNA-binding protein Roquin result in a cellintrinsic expansion of both GC B cells and $T_{F H}$ cells ${ }^{29,30}$. Thus a number of regulators, many of which are rapidly induced upon lymphocyte activation and therefore are likely to be part of the early response to antigen receptor signaling, play cell-intrinsic roles in both specialized lymphocyte subsets required to mount the GC reaction - GC B cells and $T_{F H}$ cells. Interestingly, while the action of these factors in GC B cells and $T_{F H}$ cells is usually concordant in terms of overall positive or negative effects on the GC response, the exact molecular and cellular functions of these common regulators in the two cell types are often very different.

While a number of positive transcriptional regulators of the GC reaction has been identified to date, little is known about negative regulation of this process. It was previously reported that mice with 
germline deficiency for the transcription factor Bhlhe40 developed lymphoproliferation and mild autoimmunity with age $\mathrm{e}^{31}$. The disease was associated with enlarged GCs, but the mechanism behind this phenotype remained uninvestigated. A later report demonstrated that aged Bhlhe $40^{-/-}$mice exhibit decreased regulatory $T$ cell numbers ${ }^{32}$, suggesting one possible explanation for this GC phenotype. In the present study, we aimed to test if Bhlhe40 could function as a cell-intrinsic negative regulator in cells directly participating in the GC reaction. Unexpectedly, this analysis revealed that Bhlhe40 regulates both $T_{F H}$ and $G C B$ cell arms of the GC response in a cell type-specific manner. In B cells, Bhlhe40 restrained the generation of the earliest GC B cells, but not early MBCs or PBs. In activated CD4 T cells, Bhlhe40 restrained proliferation throughout the response, thus limiting $T_{F H}$ cell numbers. With age, a dysregulated GC reaction in $\mathrm{Bh} / \mathrm{he} 4 \mathrm{O}^{-/-}$mice culminated in the development of a GC B cell lymphoma. 


\section{RESULTS}

\section{$A$ B cell-intrinsic function of Bhlhe40 in restraining the $\mathrm{GC}$ reaction}

As our RNA-seq experiments (Calderón et al, submitted; also see below) demonstrated that Bhlhe4O is rapidly upregulated upon $B$ cell activation, we first aimed to test whether Bhlhe40 has a function in activated B cells. Analysis of Bhlhe40 $0^{-/-}$mice revealed an increased number and size of spontaneous GCs in Bhlhe40 knockout (KO) mice (Fig. 1a and Supplementary Fig. 1a) and demonstrated the accumulation of cells with GC B and $T_{F H}$ cell phenotypes (Fig. 1b,c and Supplementary Fig. 1b). To test if this phenotype was underlain by a cell-autonomous function of Bhlhe40 in B cells, we established and analyzed WT:Bhlhe $40^{-1-}$ mixed bone marrow (BM) chimeras. The overall frequencies of $\mathrm{B}$ and $\mathrm{T}$ cells originating from each donor closely reflected that observed in BM progenitors (Supplementary Fig. 1c), indicating that Bhlhe40 deficiency does not have a measurable impact on the overall development of $\mathrm{T}$ and $\mathrm{B}$ lymphocytes. However, the GC B cell compartment in spleens, lymph nodes and Peyer's patches of these chimeras was dominated by cells originating from the KO donor (Fig. 1d and Supplementary Fig. 1d). This phenotype was independent of the congenic marker used to identify KO cells (Fig. $1 \mathrm{~d}$ and Supplementary Fig. 1e), indicating that Bhlhe40 deficiency and not allelic variants of genes linked to Ptprc (encodes CD45) are responsible for the phenotype. No further increase in GC B cell accumulation was observed when BM from mice double deficient for Bhlhe40 and its close homolog Bhlhe41 was used to generate mixed BM

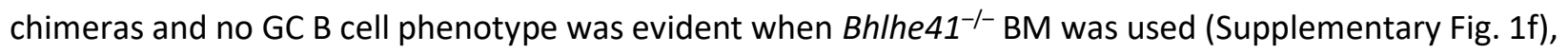
indicating that Bhlhe40, but not Bhlhe41, negatively regulates the GC reaction. As the antigen specificity of 'spontaneous' GC B cells observed in mixed BM chimeras is unclear, it was conceivable that their accumulation could have solely reflected some developmental defect (for example in tolerance formation) rather than function of Bhlhe40 in B cell activation. The increase in KO GC B cells in Peyer's patches (Fig. 1d) suggested that Bhlhe40 may restrain B cell responses to microbiota-derived antigens. To formally test if this transcription factor plays a role in the regulation of responses to foreign antigens, we 
next immunized mixed BM chimeras with phycoerythrin (PE). PE-specific GC B cells were likewise generated preferentially from KO donor B cells (Fig. 1e). We therefore conclude that Bhlhe40 restrains the $\mathrm{GC}$ reaction at least in part through its functions in activated $\mathrm{B}$ cells.

\section{Rapid transient upregulation of Bhlhe40 in activated B cells}

As a first step to unravel at which timepoint of B cell activation Bhlhe40 could execute its function, we characterized the dynamics of its expression in activated B cells. In vitro, Bhlhe 40 was rapidly (maximal level of expression 1 hour after stimulation) but transiently upregulated in B cells upon stimulation with either anti-IgM or anti-CD40 antibodies (with CD40 crosslinking resulting in the highest level of Bhlhe40 induction), suggesting that both exposure to antigen and interaction with $\mathrm{T}_{\mathrm{FH}}$ cells could be responsible for Bhlhe40 induction in vivo (Supplementary Fig. 2a). Analysis of data sets from the Immgen database ${ }^{33}$ revealed overall low Bh/he40 expression in GC B cells, with a higher level of expression in the light zone (LZ) than in dark zone (DZ) GC B cells (Supplementary Fig. 2b). As several other important regulators of the $\mathrm{GC}$ reaction, including Myc and AP4, are also expressed lowly in bulk GC B cells, but are thought to be transiently induced to high levels in a small subset of LZ GC B cells upon interaction with $T_{F H}$ cells and/or antigen $^{6,7,8}$, we next assessed Bhlhe40 expression on single-cell level by RNA flow cytometry. Indeed, Bhlhe40 was expressed by a subset of LZ GC B cells (Supplementary Fig. 2c). Bhlhe40 deficiency, however, did not affect the ratio between LZ and DZ GC B cells (Supplementary Fig. 2d). We conclude that Bhlhe40 is rapidly but transiently induced upon B cell activation and that its expression in a subset of LZ GC B cells may likewise reflect transient induction upon interaction with antigen and/or $\mathrm{T}_{\mathrm{FH}}$ cells.

\section{Bhlhe40 restricts the generation of the earliest GC B cells}

The expression analysis described above suggested that Bhlhe40 could execute its function early upon B cell activation after the initial interaction with antigen and/or $\mathrm{T}_{\mathrm{FH}}$ cells as well as later in the response, during the course of the ongoing GC reaction, through its transient induction in the LZ. To start addressing 
these possibilities, we aimed to test at which point of the response Bhlhe40-deficient B cells gain their competitive advantage. To have sufficient cell numbers for characterization of the earliest stages of the response, we crossed $B h / h e 40^{-/-}$mice to $B 1-8^{\text {hi }} \operatorname{lgh}$ knock-in mice in which B cells with $\lambda$ light chains (Ig $\lambda$ ) recognize the hapten 4-hydroxy-3-nitrophenylacetyl (NP) ${ }^{34}$. We then co-transferred congenically distinguishable Bhlhe $40^{-/-} \lg h^{B 1-8 h i /+}$ and $B h / h e 40^{+/+} \lg h^{B 1-8 h i /+}$ splenocytes (with confirmed 1:1 ratio of $\lg \lambda^{+} \mathrm{B}$ cells) into WT recipients followed by NP-OVA immunization (Fig. 2a). In line with observations made for the polyclonal responses to PE (Fig. 1e), these experiments demonstrated that the majority of donorderived GC B cells were originating from the KO donor (Fig. 2b,c). The competitive advantage of Bhlhe40deficient cells was evident for the first GC B cells that emerged around day 3 after immunization, reached its maximum by day 6 after immunization and remained stable thereafter (Fig. 2c). These results suggested that Bhlhe40 restrains the earliest stages of the GC reaction and Bhlhe40 expression in B cells may become dispensable in mature GCs. We therefore focused our attention on the events that take place during the emergence of the first GC B cells around day 3-4 after immunization. At this point, activated $\lg \lambda^{+} B 1-8^{\text {hi }}$ B cells formed three distinct populations: $C D 138^{+}$early PBs, a CCR6 ${ }^{+}$GL7 $7^{1 / \text { int }}$ population likely including non-GC-derived early MBCs as well as common precursors for all activated populations ${ }^{1,35,36}$ and CCR6-GL7 ${ }^{\text {hi }}$ early GC B cells (Fig. 2b). Strikingly, while in the competitive transfer experiments KO B1- $8^{\text {hi }}$ B cells exhibited a clear advantage in the GC B cell compartment, ratios in both PBs and in the CCR6 ${ }^{+}$ activated B cell population closely reflected the ratio in $\lg \lambda^{+} B$ cells at the time of the transfer (Fig. 2b). Of note, no difference in Bcl6 expression level was observed for WT and KO GC B cells (Supplementary Fig. 3a). The phenotype was the same when congenic markers on WT and KO B1-8 ${ }^{\text {hi }}$ B cells were inverted (Supplementary Fig. 3b). Taken together, these results demonstrated that Bhlhe40 is a highly selective negative regulator of the earliest GC B cells but not of other subsets of activated B cells.

As Bhlhe40 is a negative regulator of proliferation in several cell types ${ }^{37,38}$, we next tested if Bhlhe40 deficiency affects proliferation of GC B cells or the $\mathrm{CCR}^{+}$activated B cell population, focusing 
these experiments on the timepoint when the very first GC B cells were detectable in most of the mice. Incorporation of EdU by B1-8 ${ }^{\text {hi }}$ WT and KO B cells was comparable, and GC B cells of both genotypes exhibited much higher frequencies of $\mathrm{EdU}^{+}$cells than the $\mathrm{CCR}^{+}$population (Fig. 3a). Expectedly, no difference in EdU incorporation was detectable at later timepoints (data not shown) or in 'spontaneous' GC B cells in mixed BM chimeras (Supplementary Fig. 4a). Proliferation of in vitro activated B cells was also comparable between co-cultured WT and KO B cells across a range of anti-lgM and anti-CD40 concentrations, and the ratio between WT and KO B cells remained stable in these cultures (Supplementary Fig. 4b), suggesting that, at least in vitro, survival of B cells is also not affected by Bhlhe40 deficiency. In line with this notion, Bhlhe40 deficiency did not result in any measurable change in the frequency of cleaved Caspase 3-positive B1-8 ${ }^{\text {hi }}$ B cells in vivo, and very little apoptosis took place in GC B cells at these early timepoints irrespectively of their genotype (Fig. 3b). Likewise, apoptosis of polyclonal GC B cells was not affected by Bhlhe40 deficiency in mature GCs in mixed BM chimeras (Supplementary Fig. 4c).

The results described above suggested that Bhlhe40 is unlikely to regulate the size of the GC B cell compartment through effects on apoptosis or proliferation. We therefore hypothesized that Bhlhe40 could act through limiting the differentiation of activated cells to the GC B cell 'lineage'. To find evidence for or against this hypothesis, we next performed RNA-seq on double-sorted WT and KO CCR6 ${ }^{+}$and GC B cell populations from the B1- $8^{\text {hi }}$ co-transfer system described above at day 4 after immunization. This analysis revealed very limited changes in gene expression between WT and KO cells for both populations with less than a dozen genes significantly (adjusted $P$ value $<0.05$, fold change $\geq 2$ ) changing their expression in the KO cells (Supplementary Fig. 5a). None of these genes could be obviously connected to the phenotype. Unexpectedly, while CCR6 ${ }^{+}$cells expressed Bhlhe40, virtually no expression was observed in these early GC B cells (Supplementary Fig. 5b), suggesting that Bh/he40 functions in precursors of early GC B cells likely within the CCR6 ${ }^{+}$activated B cell population. Indeed, gene set enrichment analysis (GSEA) 
demonstrated that a GC B cell signature gene $\operatorname{set}^{39}$ was mildly but significantly upregulated in $\mathrm{KO} \mathrm{CCR6}^{+} \mathrm{B}$ cells compared to their WT counterparts (Fig. 3c). Likewise, GSEA using top 500 genes that were expressed higher in day 4 WT GC B cells than in WT CCR6' activated B cells ('Early GC B cell signature') revealed upregulation of this gene set in the KO CCR6 ${ }^{+}$population (Fig. 3c). To test if Bhlhe40 may directly repress some of these GC B cell signature genes, we performed ChIP-seq detection of the genome-wide Bhlhe40 binding in naïve B cells stimulated in vitro for 4 hours with anti-CD40 antibody. This analysis demonstrated enrichment of the expected CTCGTG E-box motif in Bhlhe40 peaks, revealed the preferential promoter and gene body binding of Bhlhe40 (Supplementary Fig. 5c), and suggested that some of the GC B cell signature genes upregulated in the KO cells may indeed be directly repressed by Bhlhe40 (Supplementary Fig. 5d).

We next compared WT and $\mathrm{KO} \lg \lambda^{+} \mathrm{B} 1-\mathrm{-}^{\text {hi }} \mathrm{B}$ cells from days 3.5 and 4 (timepoints pooled) after immunization by single cell (sc)RNA-seq. An additional WT dataset from day 2.5 after immunization was included in the initial analysis to better define the population structure of early B cell activation and capture developmental transitions. Comparison of WT and KO cells was then performed using cells from day 3.5-4 only. As described in detail elsewhere (Glaros et al, submitted), this analysis confirmed that activated B cells at day 3.5-4 formed three distinct groups corresponding to GC B cells, PBs and the CCR6 ${ }^{+}$ population containing activated precursors and early MBCs (Supplementary Fig. 5e). Both WT and KO cells were present across all the populations, but as expected, KO cells were enriched among GC B cells (Fig. 3d). Similar to the bulk RNA-seq results, scRNA-seq revealed only minor changes in gene expression between WT and KO populations (data not shown). To test if Bhlhe40 influences GC B cell differentiation, we next ranked cells by their level of expression of GC B cell signature genes (Fig. 3d, Supplementary Fig. 5f) and assessed the ratio between WT and KO cells along this trajectory. This analysis revealed a gradual increase in the relative frequency of KO cells in the course of this early GC B cell development (Fig. 3d), indicating that Bhlhe40-deficient B cells gained a competitive advantage at the earliest steps of GC B cell 
differentiation and suggesting their possible accelerated progression along this differentiation trajectory. Taken together, these results suggest that Bhlhe40 restrains the generation of the earliest GC B cells, that Bhlhe40-deficient cells gain their competitive advantage gradually in the course of early GC B cell differentiation, and that Bhlhe40 may function through mild repression of some GC B cell program genes in early activated B cells.

\section{A T cell-intrinsic function of Bhlhe 40 restrains the $T_{F H}$ cell response}

The results described above clearly identified a cell-intrinsic function of Bhlhe40 in activated B cells. To test if increased GC activity in Bhlhe40-/- mice can be fully explained by its role in B lymphocytes, we sought to inactivate $B h / h e 40$ selectively in this cell type. To this end, we generated a new conditional allele of Bhlhe40 (Supplementary Fig. 6a) and crossed mice carrying this allele to a panel of Cre lines. Ablation of Bhlhe 40 in all hematopoietic cells with Vav1-Cre phenocopied steady-state GC B and $T_{F H}$ cell phenotypes of $B h / h e 40^{-/-}$mice, indicating that they can be fully explained by Bhlhe40 function in the hematopoietic system (Fig. 4a). Unexpectedly, while deletion of Bhlhe4O in B cells with Mb1-Cre (active from pro-B cell stage on) also resulted in a GC B and $\mathrm{T}_{\mathrm{FH}}$ cell increase, both phenotypes were markedly less pronounced

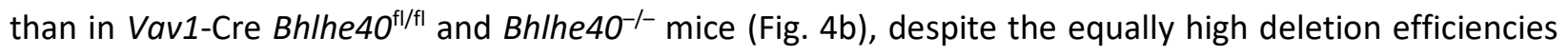
for both Cre lines in B cells (Supplementary Fig. 6b). Inactivation of Bhlhe40 later in B cell development, in immature B cells, with Cd23-Cre replicated the phenotype observed with Mb1-Cre deletion (Fig. 4c), while no phenotype was observed in Aicda-Cre Bhlhe40 $0^{\mathrm{fl} / f \mathrm{l}}$ mice (Fig. $4 \mathrm{~d}$ ). As Aicda-Cre is predominantly active in GC B cells that express the highest level of Aicda (encodes AID), these results are in line with the notion that Bhlhe40 functions in a narrow time window very early in GC B cell differentiation and that it is dispensable in mature GC B cells. The discrepancy in the magnitude of the phenotypes observed in B cell-specific (Fig. 4b,c) and pan-hematopoietic (Fig. 4a) Bhlhe40 knockouts indicated that inactivation of Bhlhe40 in another cell type in the hematopoietic system may contribute to the overall phenotype in

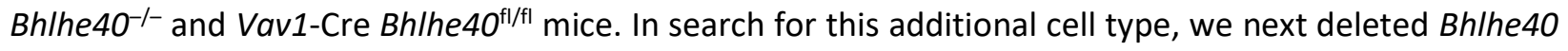


in T cells with Cd4-Cre. Strikingly, this also resulted in a measurable increase in both $\mathrm{T}_{\mathrm{FH}}$ cell and GC B cell compartments (Fig. 4e). In line with the notion that T cell help availability is a limiting factor in the GC reaction ${ }^{40}$, both phenotypes were stronger than those observed in case of B cell-specific deletion, with the $T_{F H}$ phenotype approximating that of Vav1-Cre Bhlhe $40^{f / f l}$ mice. Thus, Bhlhe40 restrains the GC reaction through its cell-intrinsic functions both in B and T lymphocytes.

We next aimed to test if Bhlhe40 executes its function upon T cell activation. In line with this possibility, Bhlhe40 was previously shown to be rapidly induced upon TCR crosslinking in naïve T cells ${ }^{32,41}$ and was expressed in $\mathrm{T}_{\mathrm{FH}}$ cells (Fig. 5a). To further characterize the function of Bhlhe40 in CD4 T cells, we

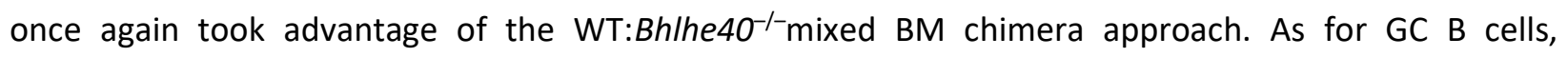
'spontaneous' $\mathrm{T}_{\mathrm{FH}}$ cells in mixed BM chimeras were preferentially generated from KO cells (Fig. 5b, Supplementary Fig. 7a). A less pronounced increase was also observed in non- $T_{F H}$ activated CD4 T cells but the frequency of $\mathrm{T}_{\mathrm{FH}}$ cells from activated $\left(\mathrm{CD} 44^{\text {hi }}{ }^{\mathrm{CD}} 62 \mathrm{~L}^{\mathrm{lo}}\right)$ cells remained higher for KO CD4 T cells (Fig. 5b). These results suggest that Bhlhe40 expression has a negative impact on the activated CD4 T cell compartment, and this impact is stronger in the case of the $T_{F H}$ cell subset. To test if this phenotype can be recapitulated in response to a foreign antigen, we next immunized mixed BM chimeras with the peptide antigens $A g 85 b_{280-294}$ or 2 W1S in complete Freund's adjuvant (CFA). Quantification of the responding cells from both donors with $\mathrm{MHC}$ class II tetramers revealed a significant increase in the frequency of antigen-specific CD4 T cells derived from the KO donor cells (Fig. 5c and Supplementary Fig. 7b) and the vast majority of these cells had a PD1 ${ }^{+} \mathrm{CXCR} 5^{+} \mathrm{T}_{\mathrm{FH}}$ cell phenotype (Fig. $5 \mathrm{C}$ ). We conclude that in addition to its function in B cells, Bhlhe40 restrains the $T_{F H}$ cell response in a cell-intrinsic manner.

\section{Bhlhe40 restrains $\mathrm{T}_{\mathrm{FH}}$ cell proliferation}

As Bhlhe40 and its close homolog Bhlhe41 were reported to have moderate anti-proliferative effects in a number of cell types ${ }^{37}$, we next tested if the competitive advantage of Bhlhe40-deficient antigen-specific 
CD4 T cells in mixed BM chimeras was underlain by their increased proliferation. Indeed, tetramer-positive KO CD4 T cells showed significantly increased EdU incorporation when compared to their WT counterparts

(Fig. 5d). RNA-seq comparison of WT and KO tetramer-positive cells revealed upregulation of signatures associated with cell cycle (G2M checkpoint, E2F target genes, and mitotic spindle) in the KO T cells (Fig. $5 e, f)$. At least some of these genes (E2f7, E2f8, Foxm1, Aurkb and many others) are likely to be directly repressed by Bhlhe40 in WT T cells as evidenced by the binding of Bhlhe40 in the proximity of these genes (Fig. $5 f$ and Supplementary Fig. 7c) in a ChIP-seq dataset from in vitro activated CD4 T cells ${ }^{42}$. More genes were upregulated than downregulated in the KO cells (73 vs 20 genes with adjusted $P$ value $<0.05$, fold change $\geq 2$ ), in line with the notion that Bhlhe40 functions predominantly as a transcriptional repressor ${ }^{37}$. Importantly, no change in the expression of known positive or negative regulators of $\mathrm{T}_{\mathrm{FH}}$ cell development and function (such as Bcl6, Prdm1, Irf4, Maf, Batf, Foxo1, or Rc3h1) was observed (Supplementary Fig. 7d). As Bhlhe40 recently emerged as an important regulator of cytokine production by different $\mathrm{T}$ cell subsets $^{42,43,44,45}$, we also checked the expression of genes encoding these cytokines in our $\mathrm{T}_{\mathrm{FH}}$-dominated antigen-specific T cells, but did not observe any changes in their expression in the KO cells (Supplementary Fig. 7d). We conclude that Bhlhe40 negatively regulates $T_{F H}$ cell expansion, at least in part through the direct repression of cell cycle-related genes.

\section{Lymphomagenesis in Bhlhe40-deficent mice}

GCs are thought to be the site of origin of the majority of mature human B cell lymphomas ${ }^{9}$. We therefore next tested if dysregulation of multiple aspects of the GC reaction in $B h / h{ }^{4} 0^{-/-}$mice could ultimately lead to lymphomagenesis. Indeed, by 20 months of age nearly all tested $B h / h e 40^{-1-}$ mice succumbed to a fatal disease manifested by the formation of ectopic lymphocyte-containing nodules in a variety of organs (Fig. 6a-c) as well as by splenomegaly. While splenomegaly was also observed in previous studies and was attributed to autoimmunity-associated lymphoproliferation ${ }^{31,32}$, ectopic lymphocyte nodules were not reported to date, likely because younger mice were analyzed in these earlier studies. Flow cytometry 
revealed a heterogeneous cellular composition of these nodular structures with the presence of CD4 and CD8 T cells as well as B cells (Fig. $6 \mathrm{~d}$ ). Strikingly, the majority of these B cells had a GC B cell phenotype, and a large fraction of CD4 T cells expressed markers of $\mathrm{T}_{\mathrm{FH}}$ cells (Fig. 6e). In line with their likely GC origin, both populations expressed Bcl6 (Fig. 6e), and GC B-like cells expressed Aicda (Supplementary Fig. 8a), however the GC architecture was not preserved in the nodules and FDC clusters were absent (Fig. $6 f) . \mathrm{T}_{\mathrm{FH}}{ }^{-}$ like cells in these structures were highly polyclonal (Fig. 6g), while GC B-like cells, in stark contrast to their normal WT or Bhlhe40-deficient counterparts, were dominated by single clones as evidenced by the predominant utilization of single IgH and IgK V segments revealed by RNA-seq (Fig. 6h). Interestingly, one of the two tumors subjected to RNA-seq expressed the Igha constant region while the second lymphoma expressed Ighm (Supplementary Fig. 8b). These results are consistent with a model in which dysregulation of the $\mathrm{GC}$ reaction in $\mathrm{Bh} / \mathrm{he} 4 \mathrm{O}^{-/-}$mice with age results in malignant transformation of a $\mathrm{B}$ cell clone, while an expanded $\mathrm{T}_{\mathrm{FH}}$ cell compartment may represent a component of the tumor 'stroma'. Of note, a similar cellular composition in tumors, with transformed B cells being outnumbered by $\mathrm{T}$ cells, has been reported for several human GC-derived B cell lymphomas ${ }^{46,47}$. To test if these B cells are indeed malignantly transformed and if they depend on the presence of $\mathrm{T}_{\mathrm{FH}}$-like cells, we transferred sorted $\mathrm{B}$ and $\mathrm{CD} 4 \mathrm{~T}$ cells from diseased mice separately or in combination into $\operatorname{Rag}^{-{ }^{-1}}$ recipients. These experiments demonstrated that $B$ cells were required and sufficient to transfer the disease (Fig. $6 \mathrm{i}$ ) and the presence of $\mathrm{T}_{\mathrm{FH}}$-like cells did not accelerate its progression, indicating that, at least at this late stage, the transformed GC B-like cells do not depend on T cell help. We conclude that the dysregulation of the GC reaction observed in Bhlhe40-deficient mice culminates in the formation of a GC-derived B cell lymphoma. 


\section{DISCUSSION}

An efficient immune response to pathogens and vaccines relies on the interplay between two specialized lymphocyte subsets - GC B cells and $T_{F H}$ cells - that takes place in the course of the GC reaction. Intriguingly, a number of transcriptional regulators have cell-intrinsic functions in both of these cell types. While many positive regulators of GC B cells and $T_{F H}$ cells were identified to date, little is known about the negative regulation of the GC reaction. In this study, we identified the transcription factor Bhlhe40 as a novel common negative regulator of both $B$ and $T$ cell sides of the GC reaction. Regulation of two very different cell types involved in the same process by highly overlapping sets of transcription factors seems rather unusual and reasons for this overlap remain unclear. It is interesting to note that many of these shared transcription factors, including Bhlhe40, are rapidly upregulated upon BCR and TCR signaling and therefore seem to represent a conserved early response program downstream of both antigen receptors. As the $\mathrm{GC}$ reaction as well as the events preceding formation of GCs rely on recurring antigen receptor signaling events both in GC B cells and $\mathrm{T}_{\mathrm{FH}}$ cells, such early response genes should be repeatedly induced in both subsets. Moreover, the complexity and highly dynamic nature of the GC reaction may allow to reveal relatively weak phenotypes that could be more difficult to capture in other settings - therefore resulting in the identification of factors with functions both in B and T cells even when these functions are subtle. Of note, most of the transcription factors shared by GCB and $T_{F H}$ cells are not exclusively involved in regulation of only $B$ and $T$ cell subsets participating in the GC reaction. This applies to Bhlhe40 that regulates cytokine production in a variety of $\mathrm{T}$ cell subsets $42,43,44,45$ and, together with Bhlhe41, contributes to regulation of the development and self-renewal of B-1a B cells $s^{38}$. It is also conceivable that shared regulators of the GC reaction could in some cases represent a convenient 'switch' that would allow to enhance or dampen both B and T cell sides of the GC response by the same upstream signals. Indeed, one could envision that if Bhlhe40 could be up- or down-regulated both in B and T lymphocytes, for 
example by a common cytokine, this would allow simultaneous negative or positive regulation of both main lymphocyte types involved in the GC reaction.

Although Bhlhe40 recently emerged as an important regulator of cytokine production by T cells ${ }^{42,}$ $43,44,45$, under the immunization conditions that favor $\mathrm{T}_{\mathrm{FH}}$ cell differentiation used in our study, Bhlhe40deficient antigen-specific CD4 T cells did not exhibit changes in expression of cytokine-encoding genes. We also did not observe dysregulation of expression of mitochondria-related genes observed in Bhlhe40deficient tissue-resident memory CD8 T cells ${ }^{41}$. Instead, activated Bhlhe40-deficient CD4 T cells in our experiments upregulated cell cycle-related genes and exhibited increased proliferation. This is in line with several reports that together suggest that Bhlhe40 and Bhlhe41 may have a mild cytostatic function across a variety of cellular contexts ${ }^{37}$. As we also previously observed antiproliferative effects of these transcription factors in B-1a cells ${ }^{38}$, we initially hypothesized that Bhlhe40 also restrained proliferation of early GC B cells or their immediate precursors. However, extensive in vivo and in vitro experiments failed to reveal any evidence for a cytostatic function of Bhlhe40 in activated B cells. The earliest GC B cells also exhibited remarkably low levels of apoptosis and no change in frequency of apoptotic cells was observed between WT and KO cells. Moreover, while Bhlhe40 was expressed in highly proliferative common activated precursors of early MBCs, PBs, and GC B cells, only the later population was selectively affected by Bhlhe40 deficiency. Taken together, these results suggested that Bhlhe40 negatively regulates GC B cell development. In line with this notion, analysis of GC B cell differentiation trajectory by scRNA-seq indicated that Bhlhe40-deficient B cells gained a competitive advantage at the earliest steps of GC B cell differentiation. Moreover, Bhlhe40-deficient activated B cells exhibited a mild upregulation of GC B cell signature genes, suggesting that they may be more prone to adopt the GC B cell fate. Taken together, these results suggest that Bhlhe40 restrains the generation of the earliest cells seeding the GC reaction.

The negative regulation of early GC B cell generation, revealed through loss-of-function experiments in this study, would suggest that overexpression of Bhlhe40 should interfere with the GC 
reaction. Indeed, corroborating our results reported here, the transgenic overexpression of Bhlhe40 driven by the $\mathrm{VH}$ promoter and the $\mathrm{E} \mu$ enhancer was previously shown to impair the generation of GC $\mathrm{B}$ cells upon immunization ${ }^{48}$.

Although Bhlhe40-/- B cells did not exhibit altered levels of Bcl6 expression, the GC B cell phenotype of Bhlhe40 deficiency was highly reminiscent of that observed in I $\mu$ Bcl6 transgenic mice. Activated $\mid \mu B$ Bcl6-transgenic B cells exhibited a $25 \%$ upregulation of Bcl6 protein over WT level already from the earliest stages of GC B cell differentiation ${ }^{49}$. This mild overexpression resulted in a competitive advantage of the transgenic cells selectively in the GCB cell compartment in the first days of the response but, very similarly to Bhlhe40 deficiency, did not affect fitness of the cells at later timepoints ${ }^{49}$. With age, I $\mu$ Bcl6 animals developed a lymphoma of GC B cell origin ${ }^{50}$.

Dysregulation of the GC reaction in Bhlhe40-deficient mice likewise culminated in the development of a GC B cell lymphoma. Cellular composition of these lymphomas strongly suggests that dysregulation of the GC reaction underlies the disease. However, the relative contribution of $B$ cell- and $\mathrm{T}_{\mathrm{FH}}$ cell-intrinsic aspects of the Bhlhe4O KO phenotype to lymphomagenesis as well as possible involvement of the other cell types in the process remain to be tested. For example, it seems probable that the age-dependent decrease in regulatory $\mathrm{T}$ cells observed in Bhlhe40-deficient mice ${ }^{32}$ can also contribute to the lymphomagenesis.

Lymphomas in Bhlhe40/- mice took more than a year to develop, suggesting that accumulation of additional mutations in GC B cells in the course of a dysregulated GC reaction may be required for the malignant transformation. In line with this notion, RNA-seq analysis of the tumor cells demonstrated that they express Aicda, suggesting that lymphoma cells could continuously undergo AID-mediated mutagenesis. Expression of the Igha constant region by one of the two tumors subjected to RNA-seq suggests that the lymphoma may have originated from an IgA-switched cell from a 'chronic' GC in 
mucosal-associated lymphoid tissue. While it remains to be seen if Bhlhe40 mutations can be found in human B cell lymphomas, the unusual cellular composition of the lymphomas in Bhlhe40-deficient mice is reminiscent of certain human malignancies. The highly heterogeneous cellular composition of the nodules in diseased Bhlhe $40^{-/-}$mice contrasts with the dominance of transformed B cells in the case of human follicular lymphoma, GC B cell-like diffuse large B cell lymphoma and Burkitt's lymphoma, and the normal GC B cell phenotype of the cells is inconsistent with classical Hodgkin's lymphomas. However, the observed phenotype has intriguing similarities to human T cell/histiocyte-rich large B cell lymphoma and nodular lymphocyte-predominant Hodgkin's lymphoma, as in both conditions transformed Bcl6-positive GC B cells are outnumbered by $T$ cells ${ }^{46,47}$. Of note, the etiology of these human lymphomas is not well understood, and it remains unknown what mutations lead to malignant transformation in these cases.

Taken together, the results reported in this study identify the transcription factor Bhlhe40 as a novel cell-intrinsic negative regulator of both $B$ and $T$ cell sides of the GC reaction, expression of which is crucial to prevent lymphomagenesis. 


\section{MATERIAL AND METHODS}

\section{Mice}

All mice used in this study were maintained on the C57BL/6 genetic background. The $\operatorname{lgh}{ }^{\mathrm{B1}-8 \mathrm{hi}}{ }^{34}, \mathrm{Bh} / \mathrm{he} 4 \mathrm{O}^{-}$ I- 31, Bhlhe41 ${ }^{-/-51}$, Rag2 $^{-/-52}, \mathrm{Mb1}-\mathrm{Cre}{ }^{53}, \mathrm{Cd} 4-\mathrm{Cre}^{54}, \mathrm{Cd} 23-\mathrm{Cre}^{55}$, Aicda-Cre ${ }^{55}$ and Vav-Cre ${ }^{56}$ mice were described previously. Bhlhe $40^{\mathrm{fl} / \mathrm{fl}}$ mice were generated as described below. WT C57BL/6J mice were obtained from Janvier Labs or bred in house. Mice analyzed in this study were at least 6 weeks old and kept under specific-pathogen-free conditions. Mice were bred and maintained at the Comparative Medicine Biomedicum facility of Karolinska Institutet (Stockholm, Sweden) or at the Research Institute for Molecular Pathology (Vienna, Austria). All mouse experiments were carried out according to valid project licenses, which were approved and regularly controlled by the Swedish and/or Austrian Veterinary Authorities.

\section{Generation of $B h / h e 40^{f l / f l}$ mice}

$B h / h e 41^{f l}$ mice were generated by CRISPR-Cas9-mediated genome editing in mouse zygotes ${ }^{57}$. A solution containing two sgRNAs targeting Bhlhe40 sequences in introns 2 and 4 (Supplementary Fig. 6a), Cas9 mRNA, Cas9 protein and ssDNA repair template was injected into C57BL/6 x CBA zygotes. Correct targeting was confirmed by sequencing and the mice were backcrossed to the C57BL/6 genetic background for 4 generations before crossing them to the Cre lines.

\section{Flow cytometry}

Mouse organs were harvested, and single cell suspensions were obtained by mincing through $70 \mu \mathrm{m}$ cell strainers. For detection of antigen-specific CD4 T cells, splenic single cell suspensions were incubated with APC-conjugated I-A ${ }^{b}$ Mtb Ag85 $b_{280-294}$ and I-A $A^{b} 2$ W1S tetramers (provided by National Institutes of Health tetramer core facility) for 1 hour prior to Fc receptor blocking and further cell surface staining. 
For detection of PE-specific B cells in the polyclonal response in mice, splenic single cell suspensions were incubated in IMDM (Thermo Fisher Scientific) pH 3.0 on ice for 1 min to remove Fc receptor-bound antibodies, washed twice with PBS/2\% FCS and stained with PE (Prozyme, $20 \mu \mathrm{g} / \mathrm{ml}$ ).

RNA flow cytometry measurement of Bhlhe 40 expression was performed using the PrimeFlow RNA Assay (Thermo Fisher Scientific) according to the manufacturer's instructions. High-sensitivity Alexa Fluor 647 probes were used. Intracellular staining for cleaved caspase-3 was performed using the 5A1E rabbit monoclonal antibody, Alexa Fluor 647-labeled anti-rabbit $\mathrm{F}(\mathrm{ab})_{2}$ fragment (both from Cell Signaling Technology) and the Cytofix/Cytoperm kit (BD) according to manufacturer's instructions. Intracellular staining for transcription factors was performed using the Foxp3 staining buffer set (Thermo Fisher Scientific) according to manufacturer's instructions. Detection of cells positive for 5-Ethynyl-2'deoxyuridine (EdU) was performed using the Click-iT Plus EdU A488 or the Click-iT Plus Pacific Blue Flow Cytometry Assay Kit (Thermo Fisher Scientific) following manufacturer's instructions with minor modifications. Data were acquired on an LSR Fortessa Flow Cytometer (BD Biosciences) and analyzed with FlowJo software v.10 (BD).

\section{Flow cytometry antibodies}

For staining of murine samples, monoclonal antibodies specific for BCL6 (REA373 and K112-91), CD4 (GK1.5, RM4-8, S11), CD3e (145-2C11, 17A2, REA641), CD8 (53-6.7), PD-1 (RMP1-30, REA802, 29F.1A12), CXCR5 (2G8, REA215), CD44 (IM7), CD62L (MEL-14), CD19 (1D3, 6D5), B220 (RA3-6B2), GL7 (GL7), CD95 (JO2 and REA453), CCR6 (REA277 and 29-2L17), CD38 (90), CD138 (REA104 and 281-2), TACI (8F10), CD86 (GL1), CXCR4 (2B11), IgM (REA979 and RMM-1), IgD (11-26c2a), IgG1 (REA1017 and RMG1-1), IgL (R26-46 and JC5-1), CD21/CD35 (7E9), CD45.1 (A20, REA1179), CD45.2 (104, 104-2), IgK (187.1), Gr-1 (RB6-8C5),

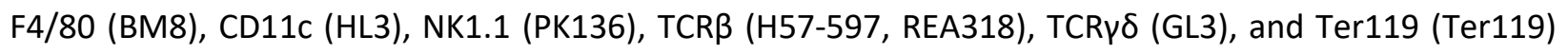
were purchased from BioLegend, BD Biosciences, Miltenyi Biotec or Thermo Fisher Scientific and were 
used at dilutions specified by the manufacturer or determined experimentally. For staining for TCR $\beta$

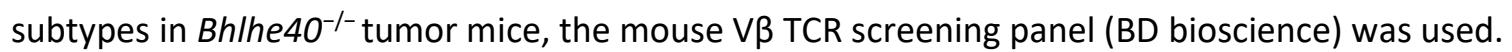

\section{Generation of mixed BM chimeras and adoptive cell transfers}

BM cells from WT and Bhlhe $40^{-/-}, B h l h e 41^{-/-}$, or Bhlhe $40^{-/-}$Bhlhe $41^{-/-}$mice were stained with $\mathrm{CD} 4, \mathrm{CD} 8$,

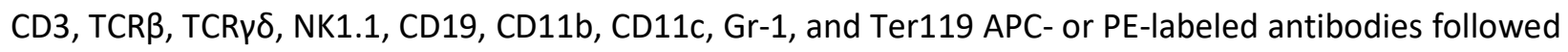
by magnetic depletion with anti-APC or -PE Micro-Beads (Miltenyi Biotec), respectively. WT and KO cells were mixed at a 1:1 ratio (unless stated otherwise) and 2-4 $\times 10^{6}$ cells were transferred intravenously into lethally irradiated (split dose -500 rads, twice) WT or Rag2 $^{-/-}$recipients (as indicated). CD45.1 and CD45.2 congenic markers were used as indicated. Chimeras were analyzed $>6$ weeks after reconstitution.

For adoptive transfer experiments, splenocytes from congenically distinguishable $B h l h e 40^{+/+} / g h^{B 1-}$ ${ }^{8 h i /+}$ and $B h / h e 40^{-/-} / g h^{B 1-8 h i /+}$ mice were mixed targeting a 1:1 ratio of WT and Bhlhe $40^{-/-}$cells in the antigenspecific B cell compartment $\left(\mathrm{CD} 19^{+} \mathrm{Ig} \lambda^{+}\right)$. The ratio was confirmed by flow cytometric analysis of an aliquot from the mix and adjusted when necessary. $10-14 \times 10^{6}$ of splenocytes $\left(\sim 3-5 \times 10^{5}\right.$ NP-specific B cells counting both donors together) were injected into the tail vein of congenically distinguishable sexmatched C57BL/6 recipient mice. Recipient mice were immunized 12-24 hours after transfer as described below.

For adoptive transfer of $\mathrm{B}$ and $\mathrm{T}$ cells from $\mathrm{Bh} / \mathrm{he} 4 \mathrm{O}^{-/-}$tumor mice, $\mathrm{CD} 19^{+} \mathrm{B}$ cells and $\mathrm{CD} 4^{+} \mathrm{T}$ cells were sorted from the spleens of sick Bhlhe $40^{-/-}$tumor mice using a Sony SH800 Cell Sorter. $>5 \times 10^{5}$ of B and/or T cells were transferred intravenously into congenically distinguishable sex-matched Rag2 $^{-/-}$ recipient mice via injection into the tail vein.

\section{Immunizations experiments}

For $\mathrm{B} 1-8^{\text {hi }}$ transfer experiments, $\mathrm{C} 57 \mathrm{BL} / 6$ recipient mice were pre-immunized by intraperitoneal injection of $100 \mu \mathrm{g}$ OVA (BioSearch Technologies) dissolved in PBS and precipitated in alum (Sigma-Aldrich) at a 1:1 
ratio $>2$ weeks prior to adoptive cell transfer. 12 to 24 hours after B1-8 ${ }^{\text {hi }}$ cell transfer, recipient mice were

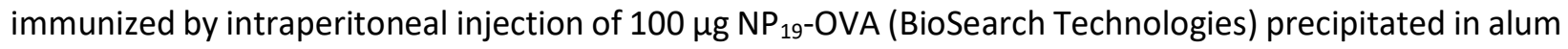
(Sigma-Aldrich) at a 1:1 ratio. For in vivo proliferation analysis, $1 \mathrm{mg}$ 5-Ethynyl-2'-deoxyuridine (EdU, Sigma-Aldrich) diluted in PBS was intraperitoneally or intravenously injected at indicated time-points post immunization. For Phycoerythrin (PE) immunization, mice were subcutaneously injected with $50 \mu \mathrm{l}$ of an emulsion containing $50 \mu \mathrm{g}$ of PE. Emulsions were prepared by mixing PE in PBS in a 1:1 ratio with Complete Freund's Adjuvant (Sigma-Aldrich). For peptide immunizations, mice were intraperitoneally injected with

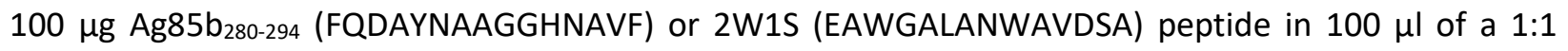
emulsion with Complete Freund's adjuvant (Sigma-Aldrich).

\section{Quantitative RT-PCR (qRT-PCR)}

WT splenic naïve B cells were isolated using CD43 Micro-Beads (Miltenyi Biotec) and stimulated with antiCD40 (1 $\mu \mathrm{g} / \mathrm{ml}$, Invitrogen) or anti-IgM (1 $\mathrm{gg} / \mathrm{ml}$, Jackson ImmunoResearch) antibody alone or in combination with IL-4 ( $20 \mathrm{ng} / \mathrm{ml}$, Peprotech) for up to 1 day. Total RNA was isolated from cultured B cells at indicated timepoints using the Quick-RNA Microprep Kit (Zymo Research). cDNA was synthesized with the RevertAid RT Kit (Thermo Scientific) and qRT-PCR was performed on a CFX96-C1000 Thermo Cycler (Bio-Rad) using the SensiFAST SYBR No-ROX Kit (Bioline, Meridian Bioscience). Hprt1 was used as a housekeeping gene and the standard curve method was used for quantification. The following primers were used: Bhlhe40-F CTC CTA CCC GAA CAT CTC AAA C, Bhlhe40-R CCA GAA CCA CTG CTT TTT CC, Hprt1 -F AGT GTT GGA TAC AGG CCA GAC, Hprt1-R CGT GAT TCA AAT CCC TGA AGT.

\section{H\&E stainings}

Tissue samples of liver and lung from $B h / h{ }^{4} \mathrm{O}^{-/-}$tumor mice were processed using the standard tissue protocol on Automatic Tissue Processor Donatello (Diapath) and paraffin-embedded. Then $2 \mu \mathrm{m}$ paraffin sections were cut. Sections were rehydrated with xylene substitute (Thermo Scientific) and ethanol, 
followed by staining with hematoxylin and eosin (both Thermo Scientific) for 1 min each and again dehydrated with ethanol and xylene substitute. After embedding in aqueous (Eukitt Neo) mounting medium, slides were analyzed using a Pannoramic FLASH 250 III slide scanner equipped with an Adimec Quartz Q12A180 camera.

\section{Immunofluorescence microscopy}

For confocal immunofluorescence microscopy, organs were harvested, fixed in 4\% PFA in PBS for 1 to 2 hours and subsequently incubated in $30 \%$ sucrose in PBS for up to 3 hours. Organs were embedded in Tissue Tek Optimal Tissue Cutting Temperature medium (OCT, Sakura Finetek) and snap frozen on dry ice. $8 \mu \mathrm{m}$ sections were cut using a cryostat and sections were mounted on SuperFrost Plus glass slides (ThermoFisher Scientific). Sections were incubated in ice-cold acetone for 10 min prior to blocking in 5\% BSA in PBS. To block endogenous biotin, the streptavidin/biotin blocking kit (Vector Laboratories) was used according to manufacturer's instructions. Staining was performed for 1 hour at RT and slides were mounted with Fluoromount Aqueous Mounting Medium (Sigma-Aldrich). Confocal images were acquired on an LSM 700 system (Carl Zeiss) with 405-, 488-, 555-, and 639 nm excitation lines at the Biomedicum Imaging Core at the Karolinska Institute. Images were processed using the ZEN 2.3 Black Edition (Carl Zeiss) or Imaris (Bitplane) Imaging software.

To analyze the size of the GCs in spleen sections of untreated WT and $B h / h e 40^{-/-}$mice, samples were prepared and imaged as described above. Additional images were acquired using a Pannoramic 250 Flash Slide Scanner equipped with a x20 objective (3DHISTECH). GCs were identified as IgD-negative areas within B cell follicles and the CaseViewer (3DHISTECH) or ImageJ software were used to measure the size of the GCs. 


\section{ChIP-seq analysis of Bhlhe40 binding}

WT splenic naïve B cells were isolated using CD43 Micro-Beads (Miltenyi Biotec) and stimulated with 1 $\mu \mathrm{g} / \mathrm{ml}$ anti-CD40 antibody (Invitrogen) for 4 hours. Cells were fixed with 1\% PFA in PBS in the presence of 1\% FCS at room temperature. Fixation was stopped after 10 minutes by addition of glycine in PBS (final concentration 0.1 M). Cells were washed with 0.1 M glycine solution in PBS and pellets were frozen. ChIP and library preparation was performed as recently described ${ }^{58}$ with minor modifications. $3 \times 10^{6}$ and $22 \times 10^{6}$ fixed frozen B cells were thawed at room temperature and diluted with SDS lysis buffer $(50 \mathrm{mM} \mathrm{Tris} / \mathrm{HCl}$ pH8, $0.5 \%$ SDS and 10mM EDTA pH8) containing $1 x$ cOmplete protease inhibitor. Cells were sonicated in a Bioruptor Plus sonicator (Diagenode). To neutralize the SDS, Triton X100 was added to a final concentration of $1 \%$ along with complete protease inhibitor. Samples were incubated at room temperature for 10 minutes. $3 \mu \mathrm{g}$ of rabbit polyclonal anti-Bhlhe40 antibody (Novus Biologicals, NB1001800, lot C2) was added to $10 \mu$ Protein G-coupled Dynabeads (Thermo Fisher Scientific) in PBS with 0.5\% BSA and incubated with rotation for 4 hours at $4^{\circ} \mathrm{C}$. Antibody-coated Dynabeads were washed with PBS with $0.5 \%$ FCS and mixed with cell lysate in PCR tubes. Tubes were incubated rotating overnight at $4^{\circ} \mathrm{C}$. Immunoprecipitated chromatin was washed with $150 \mu$ l of low-salt buffer $(50 \mathrm{mM}$ Tris/HCl, $150 \mathrm{mM} \mathrm{NaCl}$, 0.1\% SDS, $0.1 \%$ Sodium Deoxycholate, $1 \%$ Triton X-100, and $1 \mathrm{mM} \mathrm{EDTA})$, high-salt buffer (50 mM Tris/HCl, $500 \mathrm{mM} \mathrm{NaCl}, 0.1 \%$ SDS, $0.1 \%$ Sodium Deoxycholate, $1 \%$ Triton X-100, and $1 \mathrm{mM}$ EDTA) and LiCl buffer (10 mM Tris/HCl, $250 \mathrm{mM} \mathrm{LiCl,} \mathrm{0.5 \%} \mathrm{IGEPAL} \mathrm{CA-630,} \mathrm{0.5 \%} \mathrm{Sodium} \mathrm{Deoxycholate,} \mathrm{and} 1 \mathrm{mM}$ EDTA), followed by two washes with TE buffer (10 mM Tris/HCl and $1 \mathrm{mM} \mathrm{EDTA}$ ) and two washes with ice-cold Tris/ $\mathrm{HCl} \mathrm{pH} \mathrm{8.} \mathrm{For} \mathrm{tagmentation,} \mathrm{bead} \mathrm{bound} \mathrm{chromatin} \mathrm{was} \mathrm{resuspended} \mathrm{in} 30 \mu$ of tagmentation buffer. $1 \mu \mathrm{l}$ of transposase (Nextera, Illumina) was added and samples were incubated at $37^{\circ} \mathrm{C}$ for 10 minutes followed by two washes with low-salt buffer. Bead bound tagmented chromatin was diluted in $20 \mu$ of water. $25 \mu$ I PCR master mix (Nextera, Illumina) and $5 \mu$ indexed amplification primers ${ }^{59}$ (0.125 $\mu \mathrm{M}$ final concentration) were added and libraries prepared using the following PCR program: $72^{\circ} \mathrm{C} 5$ minutes 
(adapter extension); $95^{\circ} \mathrm{C} 5$ minutes; followed by 11 cycles of $98^{\circ} \mathrm{C} 10$ seconds, $63^{\circ} \mathrm{C} 30$ seconds and $72^{\circ} \mathrm{C}$ 3 minutes. All steps from sonication to library amplification PCR were performed in the same PCR tube. After PCR amplification, library cleanup was done using Agencourt AmPureXP beads (Beckman Coulter) at a PCR mix to bead ratio of 1:1. DNA concentrations in purified samples were measured using the Qubit dsDNA HS Kit (Invitrogen). Libraries were subjected to Illumina deep sequencing (NextSeq 500).

\section{Analysis of ChIP-seq data}

Bhlhe40 ChIP-seq reads from our activated B cell dataset $\left(3 \times 10^{6}\right.$ and $22 \times 10^{6} \mathrm{~B}$ cells) as well as a published activated CD4 T cell ChIP-seq dataset (GSE113054 in GEO database) ${ }^{42}$ were aligned to the mouse genome assembly version of July 2007 (NCBI37/mm9), using the Bowtie2 program ${ }^{60}$ (usegalaxy.eu; galaxy version 2.3.4. ${ }^{61}$ ) and bam files for $3 \times 10^{6}$ and $22 \times 10^{6}$ cell samples were merged. Peaks were called with a $P$ value of $<10^{-10}$ by using the MACS program version 1.3.6.1 ${ }^{62}$ with default parameters. The identified peaks were then assigned to target genes as described ${ }^{63}$. Bhlhe40 peaks overlapping with the transcription start site (TSS) were referred to as promoter peaks. For motif discovery, we used the MEME-ChIP suite (usegalaxy.eu; galaxy version $\left.4.11 .2^{61}\right)^{64}$ to predict the most significant motifs present in the $300 \mathrm{bp}$ centered at the peak summit of the top 500 sequences, as sorted by the fold enrichment score of the MACS program.

\section{RNA-seq analysis}

For RNA-seq analysis of activated B cell populations, congenically distinguishable WT and KO B1-8 ${ }^{\text {hi }}$ B cells were co-transferred into OVA-preimmunized WT recipients as described above and isolated at day 4 after NP-OVA immunization. Splenocytes were depleted using Igk/CD4/CD8/GR1/Ter119/TCRb/TCRgd/CD11b/CD11C/NK1.1 antibodies and anti-PE Micro-Beads (Miltenyi Biotec), and WT (CD45.1 $\left.1^{+} \mathrm{CD} 45.2^{-}\right)$and $\mathrm{KO}\left(\mathrm{CD} 45.1^{+} \mathrm{CD} 45.2^{+}\right)$antigen-specific B cells (CD19+Ig $\left.\lambda^{+}\right)$ GC B cells (CCR6-GL7 ${ }^{\text {hi }}$ ) or non-GC B activated B cells (CCR6 $\left.{ }^{+} G L 7^{\text {int }}\right)$ were double sorted. For the second 
sort, 500 cells were sorted directly into lysis buffer and subjected to the SMART-Seq v2 Cell protocol ${ }^{65}$. Libraries were subjected to Illumina deep sequencing (HiSeqV4 SR50).

For RNA-seq analysis of activated T cells, WT and Bhlhe $40^{-/-}$mixed BM chimeras were generated as

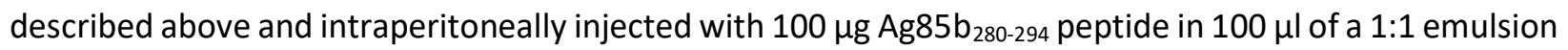
with Complete Freund's adjuvant (Sigma-Aldrich). On day 11 post immunization, spleens were harvested and single cell suspensions were incubated with APC-conjugated I-A ${ }^{\mathrm{b}} \mathrm{Mtb}$ Ag85 $\mathrm{b}_{280-294}$ tetramer (provided by National Institutes of Health tetramer core facility) for 1 hour at $4^{\circ} \mathrm{C}$ and tetramer-positive cells were enriched using anti-APC Micro-Beads (Miltenyi Biotec). CD19-CD11c CD11b ${ }^{-}$F4/80-CD8a- WT $\left(\mathrm{CD} 45.1^{+} \mathrm{CD} 45.2^{+}\right)$and Bhlhe40 ${ }^{--}\left(\mathrm{CD} 45.1^{+}\right)$antigen-specific T cells (Tetramer $\left.{ }^{+} \mathrm{CD}^{+}{ }^{+} \mathrm{TCRb}^{+}\right)$were double sorted. For the second sort, 1000 cells were directly sorted into single cell lysis solution (Invitrogen) according to manufacturer's instructions. cDNA and second strand synthesis was performed using NEBNext Ultra II RNA First Strand Synthesis (NEB cat E7771) and NEBNext Ultra II Directional RNA Second Strand Synthesis (NEB Cat E7550) modules. QIAseq FastSelect rRNA HMR kit (Qiagen) was utilized to minimize rRNA contribution to the final library. Custom made Tn5 (transposase) in combination with oligo replacement and PCR amplification was utilized to generate indexed sequencing libraries ${ }^{66}$. Libraries were pooled and paired-end sequenced (41 cycles) using the NextSeq 500 system (Illumina).

Tumor B cells from indicated organs were sorted as $\mathrm{CD} 19^{+} \mathrm{CD} 95^{+} \mathrm{GL7} 7^{+}$(mouse 1 ) or $\mathrm{CD} 19^{+} \mathrm{CD} 95^{+}$ (mouse 2). Libraries were prepared as previously described ${ }^{38}$ with minor modifications. In brief, RNA was isolated with an RNeasy Plus Mini or Micro Kits (Qiagen), and mRNA was obtained by poly(A) selection with a Dynabeads mRNA purification kit (Thermo Fisher Scientific), followed by fragmentation by heating at $94{ }^{\circ} \mathrm{C}$ for $3 \mathrm{~min}$ (in fragmentation buffer). The fragmented mRNA was used as a template for first-strand cDNA synthesis with random hexamers and a Superscript VILO cDNA Synthesis kit (Thermo Fisher Scientific). The second-strand cDNA was synthesized with $100 \mathrm{mM}$ dATP, dCTP, dGTP and dUTP in the presence of RNase H, E. coli DNA polymerase I and DNA ligase (Thermo Fisher Scientific). Sequencing 
libraries were prepared with the NEBNext Ultra II DNA Library Prep Kit for Illumina (New England BioLabs). For strand-specific RNA-sequencing, the uridines present in one cDNA strand were digested with uracil$\mathrm{N}$-glycosylase (New England BioLabs), as described ${ }^{67}$, followed by PCR amplification with NEBNext Ultrall Q5 Master Mix (New England BioLabs). Libraries were subjected to Illumina deep sequencing (HiSeqV4 SR50). For RNA-seq analysis of polyclonal WT and Bhlhe $40^{-/-}$GC B cells LZ (CD19+CD95 $\left.5^{+} \mathrm{GL} 7^{+} \mathrm{CXCR} 4^{\text {lo }} \mathrm{CD} 86^{\text {hi }}\right)$ and DZ (CD19+CD95 $\left.{ }^{+} \mathrm{GL} 7^{+} \mathrm{CXCR} 4^{\mathrm{hi}} \mathrm{CD} 86^{\mathrm{lo}}\right) \mathrm{GC}$ B cells were sorted from non-immunized mixed $\mathrm{BM}$ chimeras and subjected to RNA-seq as discussed above for tumor cells. Tracks from DZ GC B cells are shown in Figure 6h.

\section{Bioinformatic analysis of RNA-seq data}

Sequence reads that passed the Illumina quality filtering were considered for alignment. Reads corresponding to mouse ribosomal RNAs (BK000964.1 and NR046144.1) were removed. The remaining reads were cut down to a read length of $44 \mathrm{bp}$ and aligned to the mouse transcriptome (genome assembly version of July $2007 \mathrm{NCBI} 37 / \mathrm{mm} 9$ ) using TopHat version $1.4 .1^{68}$. The calculation of RNA expression values was all based on the RefSeq database, which was downloaded from UCSC on January 10th, 2014. The annotation of immunoglobulin and T cell receptor genes was incorporated from the Ensembl release 67 69. Genes with overlapping exons were flagged and double entries (i.e. exactly the same gene at two different genomic locations) were renamed. Genes with several transcripts were merged to consensusgenes consisting of a union of all underlying exons using the fuge software (I. Tamir, unpublished), which resulted in 25,726 gene models.

For analysis of differential gene expression, the number of reads per gene was counted using HTseq version $0.5 .3^{70}$ with the overlap resolution mode set to 'union'. The data sets were analyzed using the $\mathrm{R}$ package DESeq2 version 1.2.10 ${ }^{71}$. Sample normalizations and dispersion estimations were 
conducted using the default DESeq2 settings. Transcripts per million (TPM) were calculated from RNA-seq data, as described ${ }^{72}$.

GSEA was performed using the GSEA software from the Broad Institute ${ }^{73}$. Genes were ranked on the basis of their change in expression ( $\log _{2}$ fold values) as determined by the DESeq2 package and were compared to gene sets from the MSigDB or defined gene sets from literature or our data. GC B cell signature was used from Mabbott et al ${ }^{39}$ with Bcl6 and Gcsam manually added to the signature. Early GC B cell signature was generated by pairwise comparison of our WT day 4 GC B and CCR6 ${ }^{+}$B cell datasets, using top 500 GC B cell-upregulated genes.

\section{scRNA-seq library preparation}

For single cell sequencing, WT and Bhlhe $40^{-/-}$B1- $8^{\text {hi/+ }}$ B cells were co-transferred and sorted at day 3.5 (5 mice) and day 4 (5 mice) after immunization as described above for bulk RNA-seq. Cells from day 3.5 and 4 after immunization were pooled. An additional WT sample was sorted at day 2.5 after immunization. Lineage depletion for CD4/TCRß/TCRYס/CD11C/Gr1/NK1.1/Ter119/IgK was performed using MACS antiPE Micr-beads (Miltenyi Biotec). CD19+ $\lg \lambda^{+} \mathrm{CD} 45 \cdot 1^{+} \mathrm{CD} 45 \cdot 2^{-}$(WT) and CD $19^{+} \operatorname{Ig} \lambda^{+} \mathrm{CD} 45.1^{+} \mathrm{CD} 45.2^{+}\left(B h / h e 40^{-}\right.$ ${ }^{-}$) cells were double sorted using a FACS Aria III sorter. Broad gates were applied to include PBs that may start downregulating some of these surface molecules. For day 3.5-4 samples, cells were processed using the $10 \mathrm{X}$ Genomics kit version 2 immediately after sorting. For day 2.5, cells were resuspended in cold PBS/0.04\% BSA and fixed by adding 4 volumes $100 \%$ Methanol (VWR) and incubation for $30 \mathrm{~min}$ at $-20^{\circ} \mathrm{C}$. Day 2.5 cells were stored at $-80^{\circ} \mathrm{C}$ and also processed as described ${ }^{74}$ using the $10 \mathrm{X}$ Genomics kit version 3. Cells were sequenced paired end with 75 bp read length on a NextSeq550 system (Illumina).

\section{Analysis of single cell RNA-seq data}

The sequenced 10X Chromium libraries from two samples were mapped to mm10 mouse genome and assigned to droplets with Cell Ranger software (version 3.0.1) with default parameters ${ }^{75}$. Transcriptomes 
of 7,324 WT cells with the median read coverage of 2,292 (days 2.5), 2,089 WT cells with a median read coverage of 2,507 (day 3.5-4) and 1,677 KO cells with a median read coverage of 3823 (day 3.5-4) were. Joined embedding for days 2.5 and 3.5-4 was performed with scanpy based on spliced transcripts from velocyto (version 0.17 .13$)^{76}$. First, the data were normalized and filtered with scanpy.pp.recipe_seurat workflow ${ }^{77}$. Next, UMAP embedding was performed with default parameters. Cells were clustered with louvain algorithm ${ }^{78}$ and cell populations were defined as groups of clusters identified by this algorithm. GC B cell signature was used from Mabbott et al ${ }^{39}$ with $B c / 6$ and Gcsam manually added to the signature.

\section{Statistical analysis}

Statistical analysis was performed with the GraphPad Prism 8 software. Two-tailed unpaired Student's $t$-test analysis was used to assess the statistical significance of one observed parameter between two experimental groups. Two-tailed paired Student's $t$-test analysis was used to assess the statistical significance of one observed parameter between two related groups. The statistical evaluation of the RNA-seq data is described above. 


\section{ACKNOWLEDGEMENT}

We thank G. Schmauß, M. Weninger and their colleagues for sorting by flow cytometry; A. Sommer's team at the Vienna Biocenter Support Facilities GmbH (VBCF) for Illumina sequencing; A. Kavirayani and his team for histological service; Josefine Dunst for critical reading of the manuscript. This study was supported by the Swedish Research Council (grant 2017-01118 to T.K.), Cancerfonden (grant CAN 2018/710 to T.K.), Åke Wibergs Stiftelse (grant M18-0094 to T.K.), the Austrian Science Fund (grant P28841 to T.K.), and a stipend from the Wenner-Gren Foundations (to T.K.), a stipend from the German Research Foundation DFG (RE 4264/1-1 to A.R.), Boehringer Ingelheim (M.B. and R.R.) and the European Research Council (ERC) under the European Union's Horizon 2020 research and innovation program (grant agreement 740349-PlasmaCellControl, to M.B.). We thank the National Institutes of Health tetramer core facility for the preparation of MHC tetramers.

\section{REFERENCES}

1. Taylor, J.J., Jenkins, M.K. \& Pape, K.A. Heterogeneity in the differentiation and function of memory B cells. Trends in Immunology 33, 590-597 (2012).

2. $\quad$ Mesin, L., Ersching, J. \& Victora, Gabriel D. Germinal Center B Cell Dynamics. Immunity 45, 471482 (2016).

3. Crotty, S. T Follicular Helper Cell Biology: A Decade of Discovery and Diseases. Immunity 50, 1132-1148 (2019).

4. De Silva, N.S. \& Klein, U. Dynamics of B cells in germinal centres. Nature Reviews Immunology 15, 137 (2015). 
5. Finkin, S., Hartweger, H., Oliveira, T.Y., Kara, E.E. \& Nussenzweig, M.C. Protein Amounts of the MYC Transcription Factor Determine Germinal Center B Cell Division Capacity. Immunity 51, 324336.e325 (2019).

6. Calado, D.P. et al. The cell-cycle regulator c-Myc is essential for the formation and maintenance of germinal centers. Nat Immunol 13, 1092-1100 (2012).

7. Dominguez-Sola, D. et al. The proto-oncogene MYC is required for selection in the germinal center and cyclic reentry. Nat Immunol 13, 1083-1091 (2012).

8. Chou, C. et al. The Transcription Factor AP4 Mediates Resolution of Chronic Viral Infection through Amplification of Germinal Center B Cell Responses. Immunity (2016).

9. Basso, K. \& Dalla-Favera, R. Germinal centres and B cell lymphomagenesis. Nature Reviews Immunology 15, 172 (2015).

10. Nurieva, R.I. et al. Bcl6 Mediates the Development of T Follicular Helper Cells. Science 325, 10011005 (2009).

11. Yu, D. et al. The Transcriptional Repressor Bcl-6 Directs T Follicular Helper Cell Lineage Commitment. Immunity 31, 457-468 (2009).

12. Dent, A.L., Shaffer, A.L., Yu, X., Allman, D. \& Staudt, L.M. Control of Inflammation, Cytokine Expression, and Germinal Center Formation by BCL-6. Science 276, 589-592 (1997).

13. Fukuda, T. et al. Disruption of the Bcl6 Gene Results in an Impaired Germinal Center Formation. Journal of Experimental Medicine 186, 439-448 (1997).

14. Ye, B.H. et al. The BCL-6 proto-oncogene controls germinal-centre formation and Th2-type inflammation. Nature Genetics 16, 161-170 (1997).

15. Hatzi, K. et al. BCL6 orchestrates Tfh cell differentiation via multiple distinct mechanisms. Journal of Experimental Medicine 212, 539-553 (2015).

16. Shaffer, A.L. et al. BCL-6 Represses Genes that Function in Lymphocyte Differentiation, Inflammation, and Cell Cycle Control. Immunity 13, 199-212 (2000).

17. Johnston, R.J. et al. Bcl6 and Blimp-1 Are Reciprocal and Antagonistic Regulators of T Follicular Helper Cell Differentiation. Science 325, 1006-1010 (2009). 
18. Turner, C.A., Mack, D.H. \& Davis, M.M. Blimp-1, a novel zinc finger-containing protein that can drive the maturation of B lymphocytes into immunoglobulin-secreting cells. Cell 77, 297-306 (1994).

19. Oestreich, K.J., Mohn, S.E. \& Weinmann, A.S. Molecular mechanisms that control the expression and activity of Bcl-6 in TH1 cells to regulate flexibility with a TFH-like gene profile. Nature Immunology 13, 405-411 (2012).

20. Ochiai, K. et al. Transcriptional Regulation of Germinal Center B and Plasma Cell Fates by Dynamical Control of IRF4. Immunity 38, 918-929 (2013).

21. Willis, S.N. et al. Transcription Factor IRF4 Regulates Germinal Center Cell Formation through a B Cell-Intrinsic Mechanism. The Journal of Immunology 192, 3200-3206 (2014).

22. Bollig, N. et al. Transcription factor IRF4 determines germinal center formation through follicular T-helper cell differentiation. Proceedings of the National Academy of Sciences 109, 8664-8669 (2012).

23. Betz, B.C. et al. Batf coordinates multiple aspects of B and T cell function required for normal antibody responses. Journal of Experimental Medicine 207, 933-942 (2010).

24. Ise, W. et al. The transcription factor BATF controls the global regulators of class-switch recombination in both B cells and T cells. Nature Immunology 12, 536-543 (2011).

25. Inoue, T. et al. The transcription factor Foxo1 controls germinal center B cell proliferation in response to T cell help. Journal of Experimental Medicine 214, 1181-1198 (2017).

26. Sander, S. et al. PI3 Kinase and FOXO1 Transcription Factor Activity Differentially Control B Cells in the Germinal Center Light and Dark Zones. Immunity 43, 1075-1086 (2015).

27. Dominguez-Sola, D. et al. The FOXO1 Transcription Factor Instructs the Germinal Center Dark Zone Program. Immunity 43, 1064-1074 (2015).

28. Stone, E.L. et al. ICOS Coreceptor Signaling Inactivates the Transcription Factor FOXO1 to Promote Tfh Cell Differentiation. Immunity 42, 239-251 (2015).

29. Vinuesa, C.G. et al. A RING-type ubiquitin ligase family member required to repress follicular helper T cells and autoimmunity. Nature 435, 452-458 (2005).

30. Bertossi, A. et al. Loss of Roquin induces early death and immune deregulation but not autoimmunity. Journal of Experimental Medicine 208, 1749-1756 (2011). 
31. Sun, H., Lu, B., Li, R.Q., Flavell, R.A. \& Taneja, R. Defective T cell activation and autoimmune disorder in Stra13-deficient mice. Nat Immunol 2, 1040-1047 (2001).

32. Miyazaki, K. et al. The role of the basic helix-loop-helix transcription factor Dec1 in the regulatory T cells. J Immunol 185, 7330-7339 (2010).

33. Yoshida, H. et al. The cis-Regulatory Atlas of the Mouse Immune System. Cell 176, 897-912.e820 (2019).

34. Shih, T.-A.Y., Roederer, M. \& Nussenzweig, M.C. Role of antigen receptor affinity in T cellindependent antibody responses in vivo. Nature Immunology 3, 399-406 (2002).

35. Schwickert, T.A. et al. A dynamic T cell-limited checkpoint regulates affinity-dependent B cell entry into the germinal center. The Journal of Experimental Medicine 208, 1243-1252 (2011).

36. Taylor, J.J., Pape, K.A. \& Jenkins, M.K. A germinal center-independent pathway generates unswitched memory B cells early in the primary response. The Journal of Experimental Medicine 209, 597-606 (2012).

37. Ow, J.R., Tan, Y.H., Jin, Y., Bahirvani, A.G. \& Taneja, R. Chapter Nine - Stra13 and Sharp-1, the Non-Grouchy Regulators of Development and Disease. In: Reshma, T. (ed). Current Topics in Developmental Biology, vol. Volume 110. Academic Press, 2014, pp 317-338.

38. Kreslavsky, T. et al. Essential role for the transcription factor Bhlhe41 in regulating the development, self-renewal and BCR repertoire of B-1a cells. Nat Immunol 18, 442-455 (2017).

39. Mabbott, N.A. \& Gray, D. Identification of co-expressed gene signatures in mouse B1, marginal zone and B2 B-cell populations. Immunology 141, 79-95 (2014).

40. Victora, G.D. et al. Germinal center dynamics revealed by multiphoton microscopy with a photoactivatable fluorescent reporter. Cell 143, 592-605 (2010).

41. Li, C. et al. The Transcription Factor Bhlhe40 Programs Mitochondrial Regulation of Resident CD8<sup $>+</$ sup $>$ T Cell Fitness and Functionality. Immunity 51, 491-507.e497 (2019).

42. Huynh, J.P. et al. Bhlhe40 is an essential repressor of IL-10 during <em>Mycobacterium tuberculosis</em $>$ infection. The Journal of Experimental Medicine (2018).

43. Yu, F. et al. The transcription factor Bhlhe40 is a switch of inflammatory versus antiinflammatory Th1 cell fate determination. The Journal of Experimental Medicine (2018). 
44. Lin, C.-C. et al. Bhlhe40 controls cytokine production by T cells and is essential for pathogenicity in autoimmune neuroinflammation. Nat Commun 5, 3551 (2014).

45. Kanda, M. et al. Transcriptional regulator Bhlhe40 works as a cofactor of T-bet in the regulation of IFN- $p$ production in $<$ em $>\mathrm{i}</$ em $>$ NKT cells. Proceedings of the National Academy of Sciences 113, E3394-E3402 (2016).

46. Cheng, C.L. \& O'Connor, S. T cell-rich lymphoid infiltrates with large B cells: a review of key entities and diagnostic approach. Journal of clinical pathology 70, 187-201 (2017).

47. Scott, D.W. \& Gascoyne, R.D. The tumour microenvironment in B cell lymphomas. Nature Reviews Cancer 14, 517 (2014).

48. Seimiya, M. et al. Impaired lymphocyte development and function in Clast5/Stra13/DEC1transgenic mice. European Journal of Immunology 34, 1322-1332 (2004).

49. Robinson, M.J. et al. The Amount of BCL6 in B Cells Shortly after Antigen Engagement Determines Their Representation in Subsequent Germinal Centers. Cell Reports 30, 1530 1541.e1534 (2020).

50. Cattoretti, G. et al. Deregulated BCL6 expression recapitulates the pathogenesis of human diffuse large B cell lymphomas in mice. Cancer Cell 7, 445-455 (2005).

51. Rossner, M.J. et al. Disturbed clockwork resetting in Sharp-1 and Sharp-2 single and double mutant mice. PLoS One 3, e2762 (2008).

52. Shinkai, Y. et al. RAG-2-deficient mice lack mature lymphocytes owing to inability to initiate V(D)J rearrangement. Cell 68, 855-867 (1992).

53. Hobeika, E. et al. Testing gene function early in the B cell lineage in mb1-cre mice. Proceedings of the National Academy of Sciences 103, 13789-13794 (2006).

54. Lee, P.P. et al. A Critical Role for Dnmt1 and DNA Methylation in T Cell Development, Function, and Survival. Immunity 15, 763-774 (2001).

55. Kwon, K. et al. Instructive role of the transcription factor E2A in early B lymphopoiesis and germinal center B cell development. Immunity 28, 751-762 (2008).

56. de Boer, J. et al. Transgenic mice with hematopoietic and lymphoid specific expression of Cre. European Journal of Immunology 33, 314-325 (2003). 
57. Yang, H. et al. One-Step Generation of Mice Carrying Reporter and Conditional Alleles by CRISPR/Cas-Mediated Genome Engineering. Cell 154, 1370-1379 (2013).

58. Gustafsson, C., De Paepe, A., Schmidl, C. \& Månsson, R. High-throughput ChIPmentation: freely scalable, single day ChIPseq data generation from very low cell-numbers. BMC Genomics 20, 59 (2019).

59. Buenrostro, J.D., Giresi, P.G., Zaba, L.C., Chang, H.Y. \& Greenleaf, W.J. Transposition of native chromatin for fast and sensitive epigenomic profiling of open chromatin, DNA-binding proteins and nucleosome position. Nature Methods 10, 1213 (2013).

60. Langmead, B. \& Salzberg, S.L. Fast gapped-read alignment with Bowtie 2. Nature Methods 9, 357 (2012).

61. Afgan, E. et al. The Galaxy platform for accessible, reproducible and collaborative biomedical analyses: 2018 update. Nucleic Acids Research 46, W537-W544 (2018).

62. Feng, J., Liu, T., Qin, B., Zhang, Y. \& Liu, X.S. Identifying ChIP-seq enrichment using MACS. Nature Protocols 7, 1728 (2012).

63. Revilla-i-Domingo, R. et al. The B-cell identity factor Pax5 regulates distinct transcriptional programmes in early and late B lymphopoiesis. EMBO J 31, 3130-3146 (2012).

64. Machanick, P. \& Bailey, T.L. MEME-ChIP: motif analysis of large DNA datasets. Bioinformatics 27, 1696-1697 (2011).

65. Picelli, S. et al. Full-length RNA-seq from single cells using Smart-seq2. Nature Protocols 9, 171181 (2014).

66. Gertz, J. et al. Transposase mediated construction of RNA-seq libraries. Genome Research 22, 134-141 (2012).

67. Parkhomchuk, D. et al. Transcriptome analysis by strand-specific sequencing of complementary DNA. Nucleic acids research 37, e123-e123 (2009).

68. Trapnell, C., Pachter, L. \& Salzberg, S.L. TopHat: discovering splice junctions with RNA-Seq. Bioinformatics 25, 1105-1111 (2009).

69. Cunningham, F. et al. Ensembl 2015. Nucleic acids research 43, D662-D669 (2015). 
70. Anders, S., Pyl, P.T. \& Huber, W. HTSeq-A Python framework to work with high-throughput sequencing data. Bioinformatics, btu638 (2014).

71. Love, M.I., Huber, W. \& Anders, S. Moderated estimation of fold change and dispersion for RNAseq data with DESeq2. Genome Biol 15, 550 (2014).

72. Wagner, G.P., Kin, K. \& Lynch, V.J. Measurement of mRNA abundance using RNA-seq data: RPKM measure is inconsistent among samples. Theory in Biosciences 131, 281-285 (2012).

73. Subramanian, A. et al. Gene set enrichment analysis: A knowledge-based approach for interpreting genome-wide expression profiles. Proceedings of the National Academy of Sciences 102, 15545-15550 (2005).

74. Chen, J. et al. PBMC fixation and processing for Chromium single-cell RNA sequencing. Journal of Translational Medicine 16, 198 (2018).

75. Zheng, G.X.Y. et al. Massively parallel digital transcriptional profiling of single cells. Nature Communications 8, 14049 (2017).

76. La Manno, G. et al. RNA velocity of single cells. Nature 560, 494-498 (2018).

77. Satija, R., Farrell, J.A., Gennert, D., Schier, A.F. \& Regev, A. Spatial reconstruction of single-cell gene expression data. Nature Biotechnology 33, 495-502 (2015).

78. Blondel, V.D., Guillaume, J.-L., Lambiotte, R. \& Lefebvre, E. Fast unfolding of communities in large networks. Journal of Statistical Mechanics: Theory and Experiment 2008, P10008 (2008). 


\section{FIGURE LEGENDS}

Figure 1. B cell-intrinsic function of Bhlhe40 in restraining the GC reaction. (a) Representative images of spleen sections of unchallenged WT and $B h / h e 40^{-1-}$ mice as analyzed by confocal immunofluorescence microscopy. Germinal centers (GCs), identified by GL7 (white) and CD21/CD35 (yellow) expression in IgD (red)-negative areas, are highlighted. One of two independent experiments is shown. (b,c) Quantification of flow cytometric analysis of GC B cells (b) and $\mathrm{T}_{\mathrm{FH}}$ cells (c) in unchallenged WT and $B h / h e 40^{-/-}$mice. Pooled data from two independent experiments with $n=9(\mathrm{WT})$ and $n=10$ (KO) mice (b) and $n=7$ mice for each genotype (c) are shown. Surface expression of CD95 and GL7 on CD19+ B cells (b) and surface expression of PD1 and intracellular Bcl6 in $\mathrm{CD}^{+} \mathrm{T}$ cells (c) are shown. (d,e) Mixed BM chimeras were generated by transferring a 1:1 mixture of congenically distinguishable WT (CD45.1 $)$ and $B h / h e 40^{-/-}$ $\left(\mathrm{CD} 45.2^{+}\right) \mathrm{BM}$ progenitor cells into lethally irradiated $\mathrm{Rag}^{-/-}$recipients. Mice were analyzed $>6$ weeks after transfer. (d) Flow cytometric analysis and quantification of the GC B cell population in spleen, peripheral lymph nodes (LN) and Peyer's Patches (PP) in mixed BM chimeras. One experiment with $n=6$ mice is shown, representative of at least three independent experiments. (e) Mixed BM chimeras were immunized with PE emulsified in Complete Freund's adjuvant. Antigen (PE)-specific GC B cells were analyzed by flow cytometry 21 days post immunization. GL7 expression and PE-binding on CD19+ $\mathrm{B}$ cells (left) and quantification of PE-specific GC B cells (gated as $C D 95^{+} G L 7^{+} P E^{+}$) among donor $C D 19^{+} B$ cells (right) are shown. Pooled data from three independent experiments with $n=12$ mice are shown. Data were analyzed with unpaired Student's $t$-test $(\mathbf{b}, \mathbf{c}$, mean \pm SD is shown) and paired Student's $t$-test $(\mathbf{d}, \mathbf{e})$. $* P<0.05, * * P<0.01, * * * P<0.001, * * * * P<0.0001$. One dot represents one mouse.

Figure 2. Bhlhe40 restricts the earliest stages of the GC B cell response. (a) Schematic representation of the experimental setup for b-c. OVA-primed $C D 45.2^{+}$WT mice were injected with a 1:1 mixture of congenically distinguishable WT (CD45.1 $1^{+}$and Bhlhe $40^{-/-}\left(\mathrm{CD} 45.1^{+} / \mathrm{CD} 45.2^{+}\right) \mathrm{B} 1-8^{\mathrm{hi} /+}$ splenocytes and were 
immunized with NP-OVA in alum next day. (b) (top) Surface expression of CD138, CCR6 and GL7 on $\mathrm{CD} 45.1^{+} \lg \lambda^{+} \mathrm{CD} 19^{+} \mathrm{B}$ cells (intracellular Ig $\lambda$ staining for PB gating) and quantification of WT and $\mathrm{Bh} / \mathrm{he} 40^{-I^{-}}$ -derived cells among total $C D 45.1^{+} \operatorname{Ig} \lambda^{+} \mathrm{CD} 19^{+} \mathrm{B}$ cells at the time of the transfer, as well as among plasmablasts (PB), CCR6 ${ }^{+}$and $\mathrm{GC}$ B cells on day 5 post immunization is shown. One experiment with $\mathrm{n}=4$ mice is shown, representative of three independent experiments. (c) Representative flow cytometric analysis and quantification of WT and $B h / h e 40^{-l_{-}}$-derived cells among CD45. $1^{+} \lg \lambda^{+} \mathrm{CD} 19^{+} \mathrm{CCR} 6^{+}$and GC B cells at indicated time points post immunization. Gating for total $\lg \lambda^{+} C D 19^{+} B$ cells was applied for day 0 (transfer mix) and day 2 (prior to GC B cell emergence) after immunization. The time course is based on pooled data from two independent experiments with $n=4-16$ mice for each time point. One dot represents the mean with SD for each time point.

Figure 3. Bhlhe40 restrains the generation of early GC B cells. (a-d) OVA-primed CD45.2+ WT mice were injected with a 1:1 mixture of congenically distinguishable WT $\left(\mathrm{CD} 45.1^{+}\right)$and Bhlhe40 ${ }^{-/-}\left(\mathrm{CD} 45.1^{+} / \mathrm{CD} 45.2^{+}\right)$

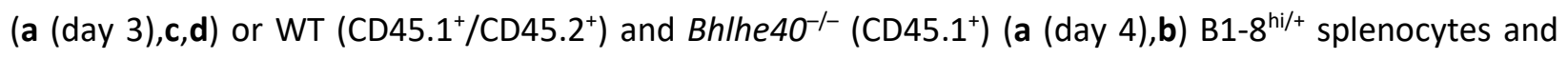
were immunized with NP-OVA in alum next day. (a,b) Flow cytometric analysis and quantification assessing the proliferation (a) and frequency of apoptotic cells (b) of WT and Bhlhe $40^{-/-}$CCR6 $6^{+}$and GC B cells on day $3(\mathbf{a})$ and $4(\mathbf{a}, \mathbf{b})$ post immunization. (a) EdU was i.v. injected 4 and 2 hours prior to analysis. (b) Apoptotic cells were identified by intracellular staining for active caspase-3. (a) One experiment with $n=14$ (day 3 ) and $n=6$ (day 4) mice is shown. Representative of at least two independent experiments. (b) Pooled data from two independent experiments with $n=10$ mice are shown. Note that only in 2 mice out of 10 active caspase- $3^{+}$cells were detectable in the GC B cell compartment. Flow cytometry plots show electronically merged data from 3 mice, and mean \pm SD is shown. Data were analyzed with paired Student's $t$-test. $* P<0.05$. One dot represents one mouse. (c) Left - Gene set enrichment analysis (GSEA) on an RNA-seq dataset ranked by KO/WT fold change in day $4 \mathrm{CCR}^{+} \mathrm{B} 1-8^{\text {hi }} \mathrm{B}$ cells using a published GC B cell signature ${ }^{39}$ ('GC B cell signature') or the top 500 genes upregulated in WT day 4 B1-8 ${ }^{\text {hi }}$ GC B cells when 
compared to WT day 4 CCR6 ${ }^{+}$B cells ('Early GC B cell signature') as gene sets. NES, normalized enrichment score; FDR, false-discovery rate. Right - examples of genes that were enriched in KO CCR6 ${ }^{+} \mathrm{B} 1-8^{\text {hi }} \mathrm{B}$ cells as identified by GSEA. Expression in WT and Bhlhe $40^{-/-}$CCR6 ${ }^{+}$B cells as assessed by RNA-seq analysis on day 3.5-4 post immunization is shown. (d) scRNA-seq comparison of WT and KO GC B cell differentiation. Left - UMAP plots showing the distribution of WT (days 2.5 and/or 3.5-4 after immunization) and KO (days 3.5-4 after immunization) B1-8 ${ }^{\text {hi }}$ B cells. Middle - UMAP plot showing the expression of GC B cell signature genes in clusters that were included for the subsequent WT/KO comparison: the frequency of WT (blue) and KO (red) B cells among cells binned by the strength of expression of GC B signature genes is shown.

Figure 4. Bhlhe 40 restrains the GC reaction through its cell-intrinsic functions in both B and T cells. Flow cytometric analysis and quantification of GC B cells and $\mathrm{T}_{\mathrm{FH}}$ cells in unchallenged (a) WT, Bhlhe40 ${ }^{-/-}$and Vav1-Cre Bhlhe $40^{\mathrm{fl} / f \mathrm{l}}$ mice, (b) Bhlhe $40^{\mathrm{fl} / \mathrm{fl}}$ and $\mathrm{Mb1}$-Cre Bhlhe $40^{\mathrm{fl} / \mathrm{fl}}$ mice, (c) Bh/he $40^{\mathrm{fl} / f \mathrm{l}}$ and $\mathrm{Cd} 23-\mathrm{Cre}$ Bh/he $40^{\mathrm{fl} / \mathrm{fl}}$ mice, (d) Bh/he $40^{\mathrm{fl} / \mathrm{fl}}$ and Aicda-Cre Bh/he $40^{\mathrm{fl} / f \mathrm{fl}}$ mice, (e) Bh/he $40^{\mathrm{fl} / f \mathrm{fl}}$ and Cd4-Cre Bh/he $40^{\mathrm{fl} / f \mathrm{l}}$ mice. Pooled data from three independent experiments with (a) $n=24\left(\right.$ WT) $, n=27\left(\right.$ Bh/he $\left.40^{-/-}\right), n=14($ Vav1Cre Bhlhe $\left.40^{f / / f l}\right),\left(\right.$ b) $n=10\left(B h / h e 40^{f / / f}\right)$ and $n=19\left(M b 1-C r e B h / h e 4 O^{f / / f l}\right),(\mathbf{c}) n=12\left(B h / h e 40^{f / / f}\right)$ and $n=26$

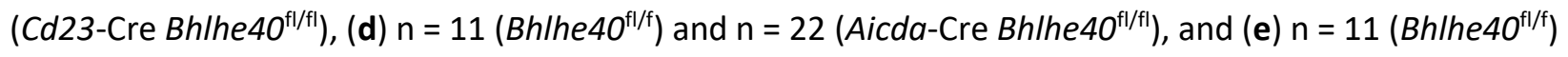
and $\mathrm{n}=16\left(\mathrm{Cd} 4-\mathrm{Cre} B\right.$ h/he $\left.40^{\mathrm{fl} / \mathrm{fl}}\right)$ mice are shown. Fold changes are indicated. Mean values are shown with SD and data were analyzed with Student's $t$-test. One dot represents one mouse. ${ }^{*} P<0.05, * * P<0.01$, $* * * P<0.001, * * * * P<0.0001$.

Figure 5. Bhlhe40 limits the proliferation of $\mathrm{T}_{\mathrm{FH}}$ cells. (a) Bhlhe40 expression in $\mathrm{T}_{\mathrm{FH}}$ cells among $\mathrm{CD}^{+} \mathrm{T}^{\mathrm{T}}$ cells from spleens of unchallenged WT mice as analyzed by RNA flow cytometry. One experiment with pooled data from $n=2$ mice is shown. Representative of two independent experiments. (b) Mixed BM chimeras were generated by transferring a 1:1 mixture of congenically distinguishable WT $\left(C D 45.1^{+}\right)$and Bhlhe $40^{-/-}\left(\mathrm{CD} 45.2^{+}\right) \mathrm{BM}$ progenitor cells into lethally irradiated $\mathrm{Rag}^{-{ }^{--}}$recipients. Mice were analyzed > 6 weeks after transfer. Expression of PD1 and Bcl6 by WT and Bhlhe $40^{-1-}$ Foxp3 $^{-} \mathrm{CD}^{+} \mathrm{T}$ cells (top row left), 


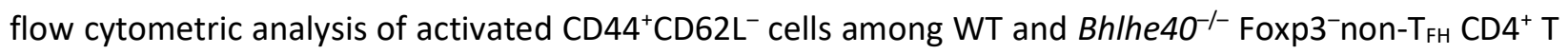
cells (top row right), and quantification among total or activated WT and Bhlhe $40^{-1-}$ Foxp $^{-} \mathrm{CD}^{+} \mathrm{T}^{\mathrm{T}}$ cells in the spleen (bottom row) are shown. For quantification, $\mathrm{T}_{\mathrm{FH}}$ cells were gated as Foxp3 ${ }^{-} \mathrm{Bcl}^{+} \mathrm{CD}^{+}{ }^{+} \mathrm{T}$ cells or Foxp3-Bcl6 ${ }^{+} \mathrm{PD} 1^{+} \mathrm{CD} 4^{+} \mathrm{T}$ cells (in ' $\mathrm{T}_{\mathrm{FH}}$ of donor $\mathrm{CD} 4^{+} \mathrm{T}^{\prime}$ ). One experiment with $\mathrm{n}=6$ mice is shown. Representative of two independent experiments. (c-f) Mixed BM chimeras were generated by transferring a 1:1 mixture of congenically distinguishable WT $\left(\mathrm{CD} 45.1^{+} / \mathrm{CD} 45.2^{+}\right)$and Bhlhe $40^{-{ }^{-}}\left(\mathrm{CD} 45.1^{+}\right) \mathrm{BM}$ progenitor cells into lethally irradiated WT $\left(C D 45.2^{+}\right)$recipients. Mice were immunized with $M$. tuberculosis Ag85 $\mathrm{b}_{280-294}$ peptide emulsified in Complete Freund's adjuvant $>7$ weeks after transfer. (c) Flow cytometric analysis and quantification of Ag85b-tetramer-binding $\mathrm{CD}^{+}{ }^{+} \mathrm{T}$ cells and $\mathrm{T}_{\mathrm{FH}}$ cells among WT and Bhlhe40 ${ }^{-1-} \mathrm{CD}^{+} \mathrm{T}$ cells in the spleen on day 11 post immunization. (d) Immunized mixed BM chimeras were i.p. injected with EdU 18 and 14 hours before harvest on day 11. EdU incorporation by

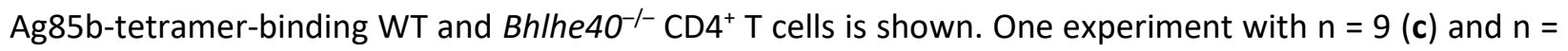
10 (d) mice is shown, respectively. Representative of two independent experiments each. Data were analyzed with paired Student's $t$-test. $* P<0.05, * * P<0.01,{ }^{* * *} P<0.001$. One dot represents one mouse. (e,f) RNA-seq analysis of Ag85b-tetramer-binding WT and Bh/he $40^{-/-} \mathrm{CD}^{+} \mathrm{T}$ cells isolated from mixed BM chimeras 11 days post immunization. (e) GSEA showing top 3 most enriched gene sets from MSigDB Hallmark collection: G2M checkpoint, E2F target and mitotic spindle genes depicting the change in their

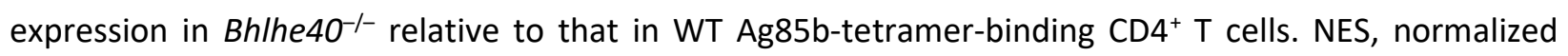
enrichment score; FDR, false-discovery rate. (f) Comparison of changes in gene expression induced by Bhlhe40 deficiency in Ag85b-tetramer-binding CD4 ${ }^{+} \mathrm{T}$ cells. $\log _{2}$-transformed Bhlhe40-/-/WT fold changes are plotted and the number of significantly $(>2$-fold, adjusted $P<0.05)$ up- and downregulated genes is indicated. Cell cycle-related genes (GO:0007049) that significantly ( $>2$-fold, adjusted $P<0.05$ ) changed their expression in Bhlhe $40^{-/-}$cells are highligted. Gene names in bold correspond to genes with assigned Bhlhe40 ChIP-seq peaks. 
Figure 6. Lymphomagenesis in ageing Bhlhe40-/- mice. (a) Image of a 15 month-old $B h / h e 40^{-1-}$ mouse demonstrating splenomegaly (arrow) and nodular structures (asterisks) in the liver. (b) Kaplan-Meier survival curve depicting the survival of ageing WT and $B h / h e 40^{-/-}$mice. Pooled data for WT $\mathrm{n}=15$ and Bhlhe $40^{-/-} \mathrm{n}=21$ mice are shown. (c) H\&E staining of sections of nodules in the liver and lung from two

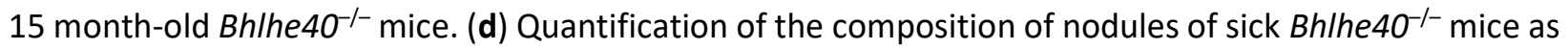
assessed by flow cytometry. (e) Flow cytometric analysis of GL7, PD1 and Bcl6 expression in the CD19+ B

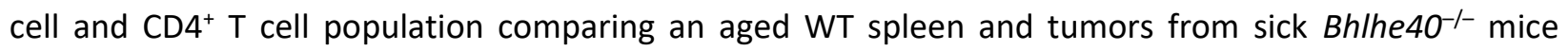
between 15 and 18 month of age. Data are representative of $n=12$ sick Bhlhe $40^{-/-}$mice. (f) Representative image of a tumor nodule section from the liver of a sick Bhlhe $40^{-/-}$mouse. CD3 (green), B220 (blue), CD21/CD35 (red) and GL7 (white) expression are shown. Data are representative of three analyzed mice.

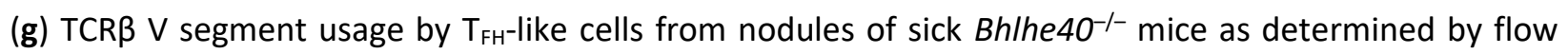
cytometry. (h) Genome browser view of V segments in the Igh and Igk locus as determined by RNA-seq analysis of GC B cell-like cells of two sick Bhlhe $40^{-/-}$mice. (i) Kaplan-Meier survival curve depicting the survival of $\mathrm{Rag}^{-{ }^{--}}$recipients that were transferred with sorted B and T cells from a sick Bhlhe $4 \mathrm{O}^{-{ }^{-}}$mouse. Pooled data from two independent experiments with $n=12$ mice for B cells, $n=9$ for T cells and $n=6$ for $\mathrm{T}+\mathrm{B}$ cells are shown. 
bioRxiv preprint doi: https://doi.org/10.1101/2021.03.12.435122; this version posted March 12, 2021. The copyright holder for this preprint (which was not certified by peer review) is the author/funder. All rights reserved. No reuse allowed without permission.

a

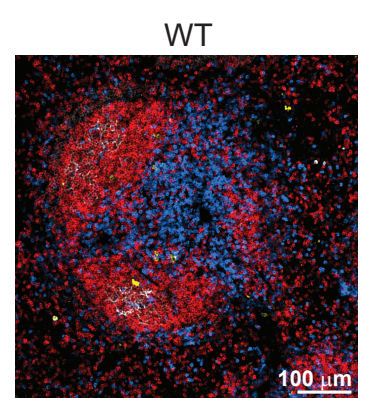

$100 \mathrm{~mm}$

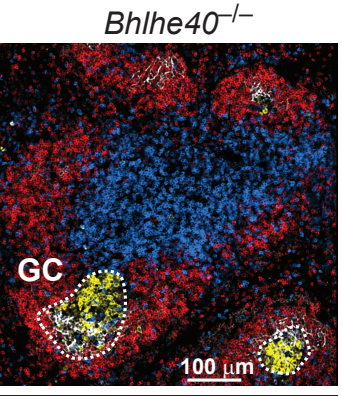

GL7

d

Mixed BM chimera

- WT (CD45.1)

- Bhlhe40-/- (CD45.2)

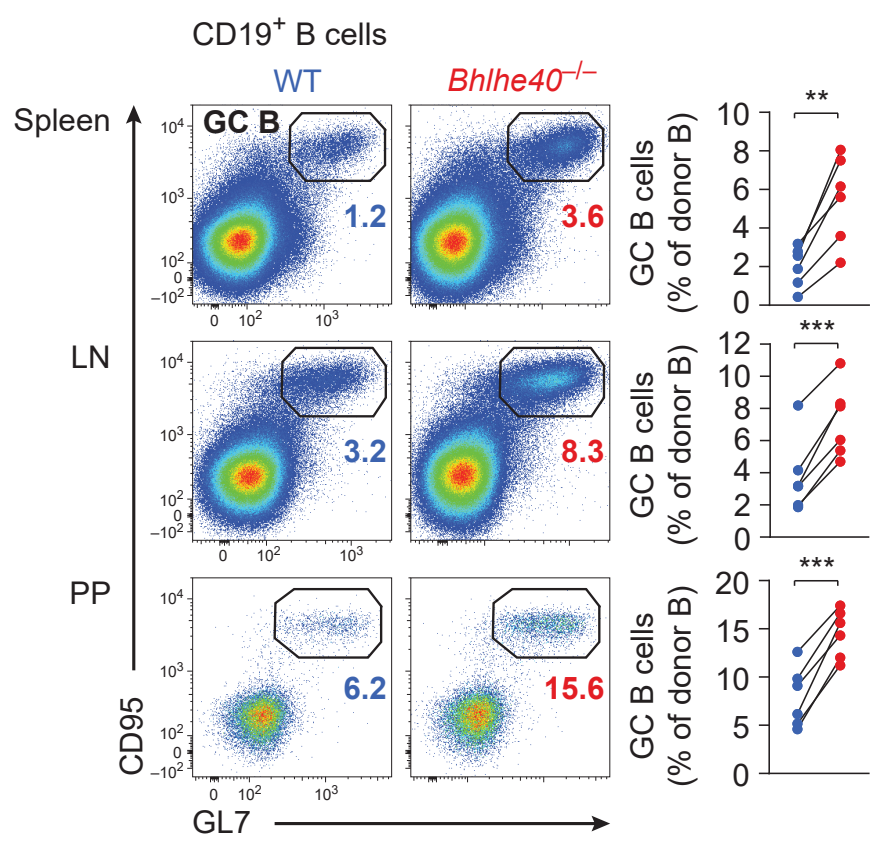

b

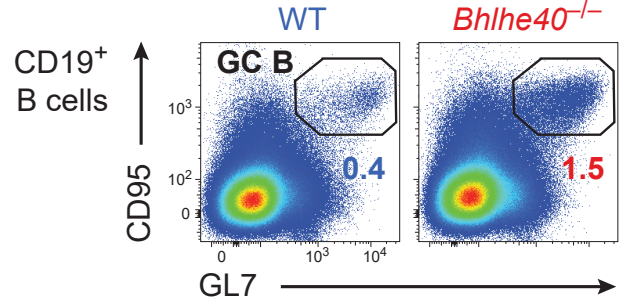

C

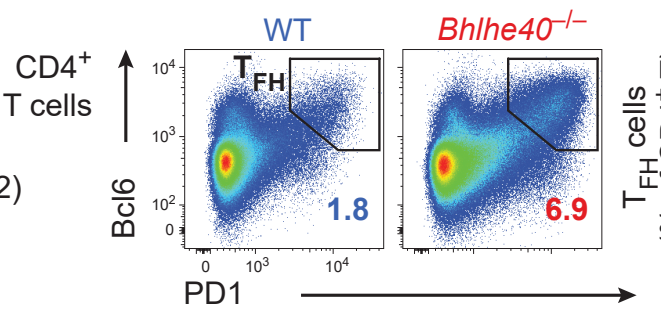

e

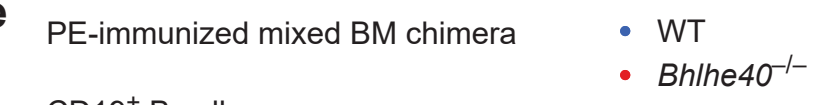

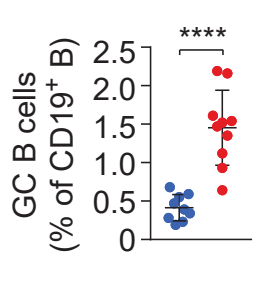

- WT

- Bhlhe40-1-
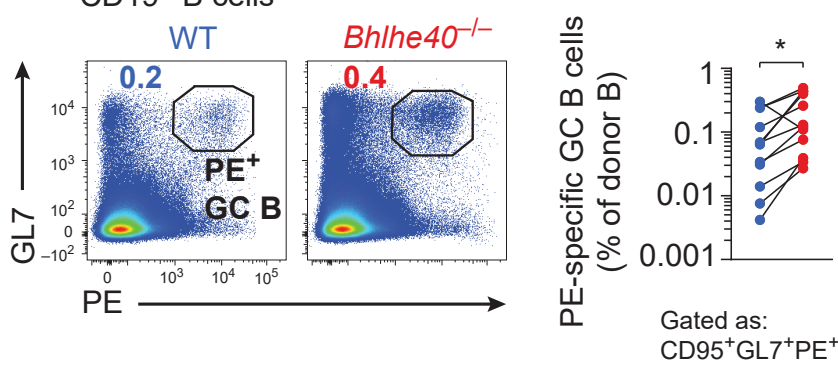
bioRxiv preprint doi: https://doi.org/10.1101/2021.03.12.435122; this version posted March 12, 2021. The copyright holder for this preprint (which was not certified by peer review) is the author/funder. All rights reserved. No reuse allowed without permission.

a

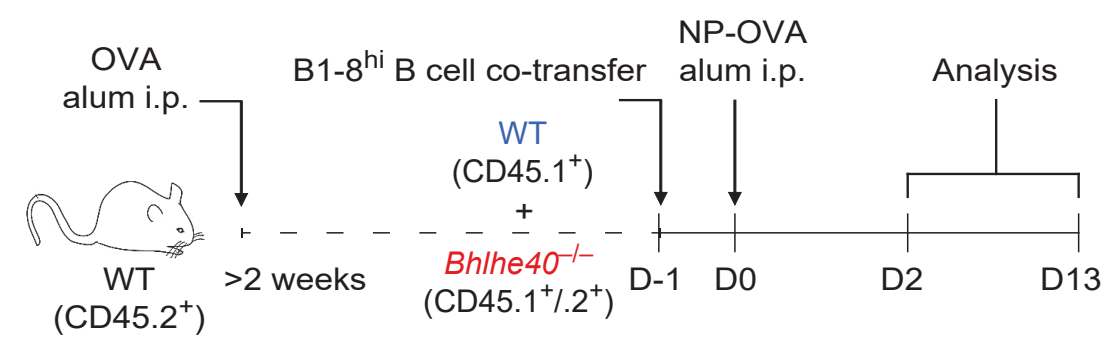

b

Day 5

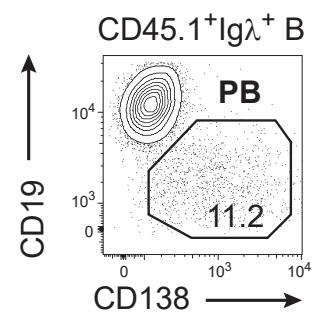

$\mathrm{CD} 45.1^{+} \lg \lambda^{+} \mathrm{B}$

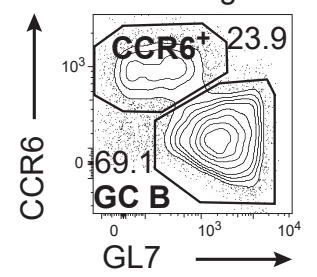

Day -1
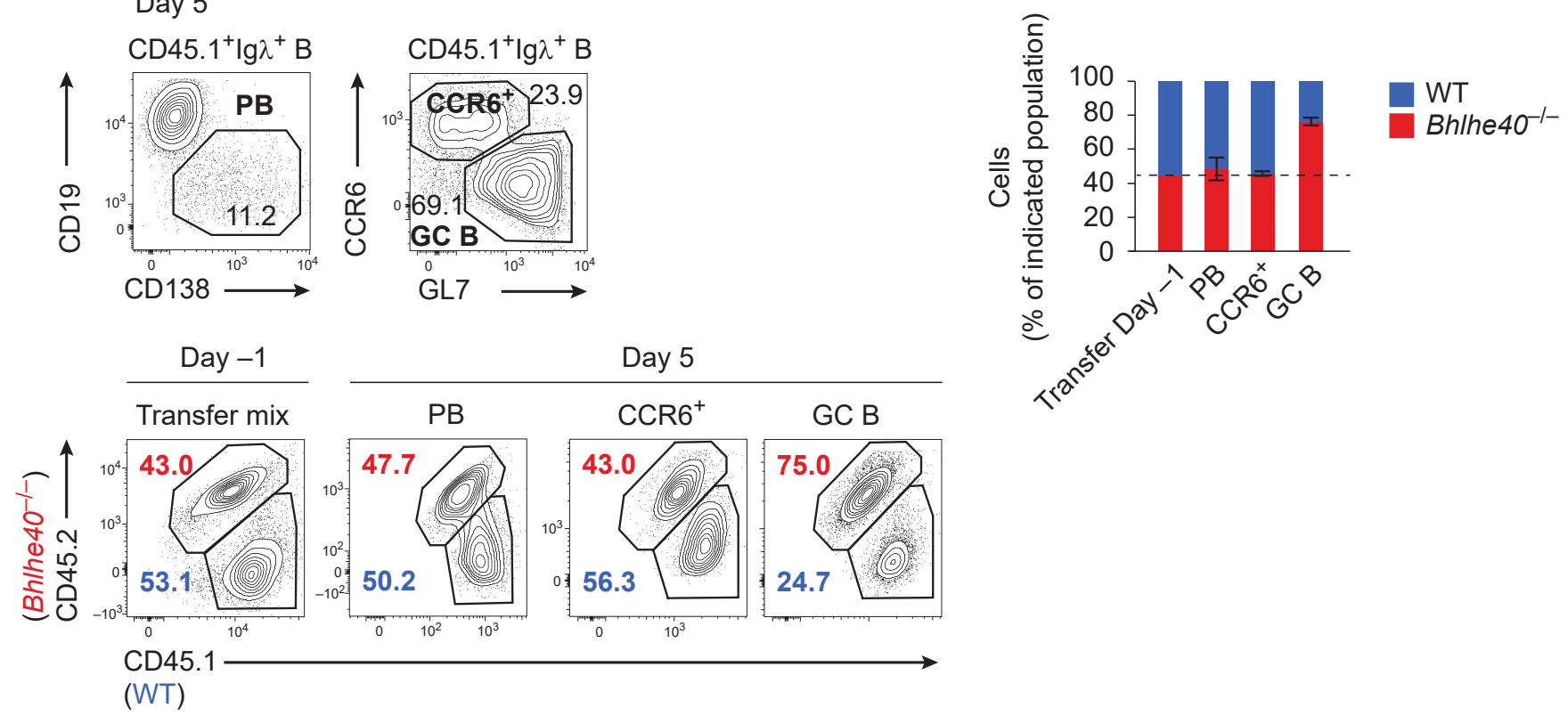

C
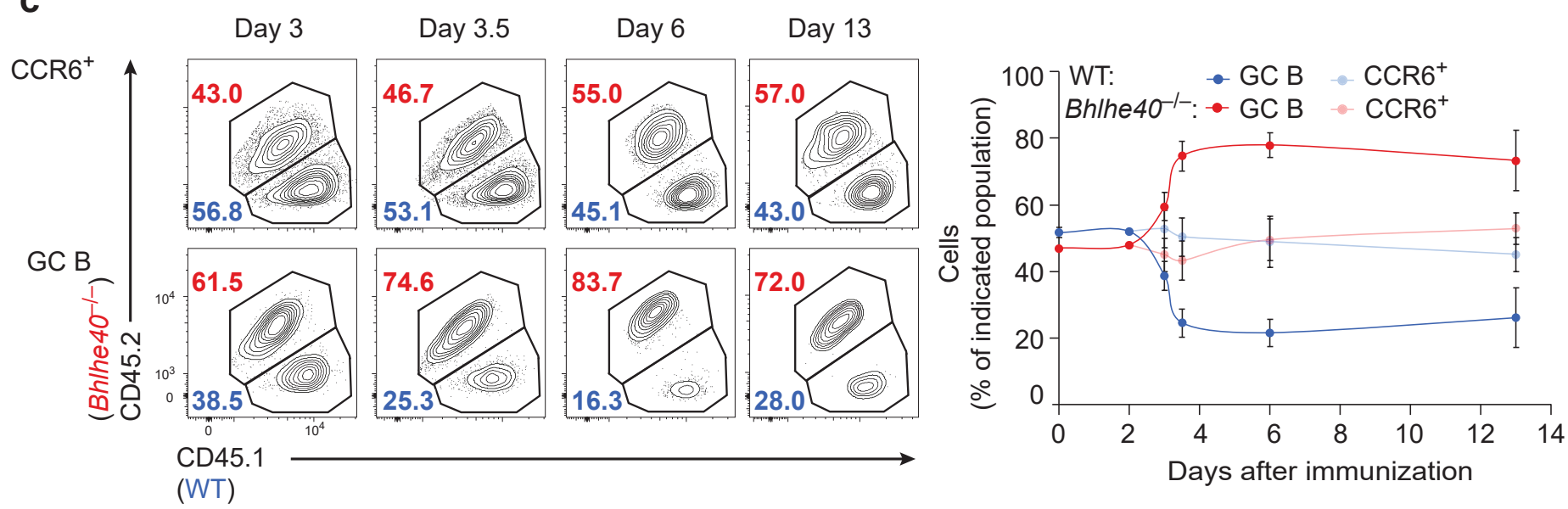

Figure 2 
bioRxiv preprint doi: https://doi.org/10.1101/2021.03.12.435122; this version posted March 12, 2021. The copyright holder for this preprint (which was not certified by peer review) is the author/funder. All rights reserved. No reuse allowed without permission.

a

B1-8 ${ }^{\text {hi }}$ co-transfer

Day 3 - WT • Bhlhe40-1-

Day 4
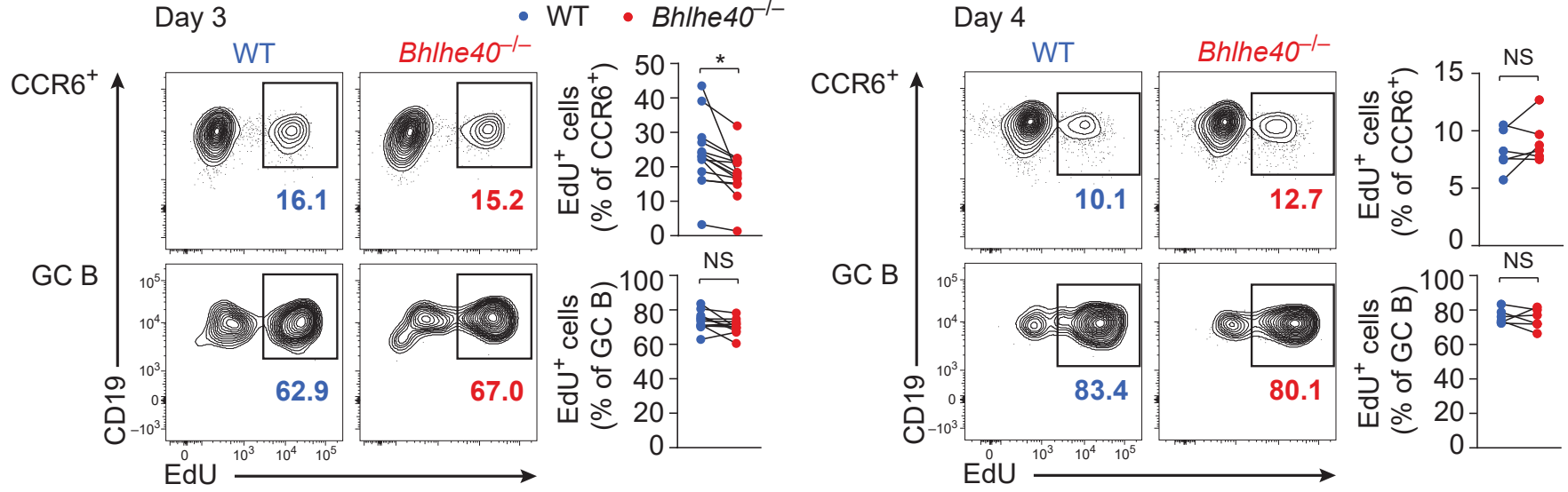

b

B1-8 ${ }^{\text {hi }}$ co-transfer

Day 4

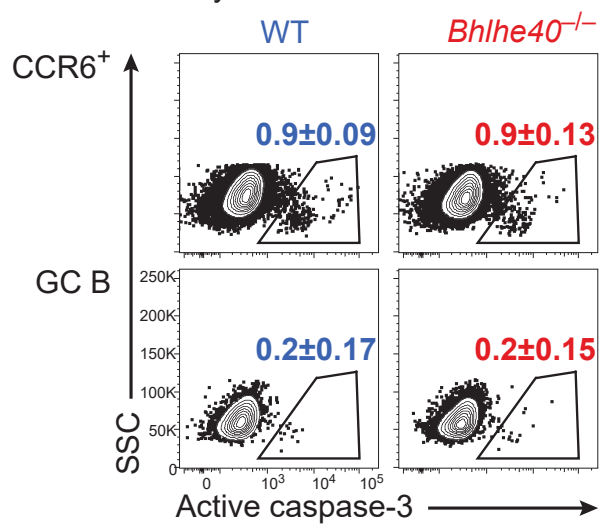

- WT - Bhlhe40-1-

C

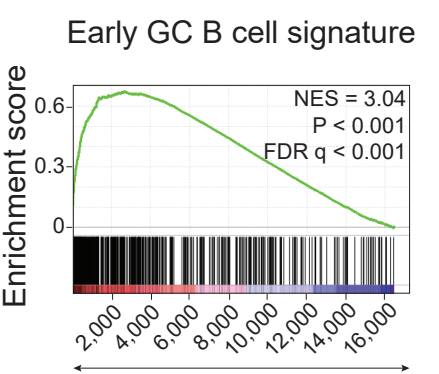

Up in Bhlhe $40^{-1-}$ CCR6 $^{+}$
Up in WT CCR6 $^{+}$

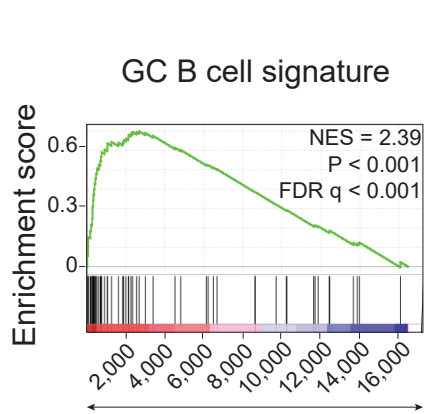

Up in Bhlhe40-/- Up in WT CCR6 $^{+}$

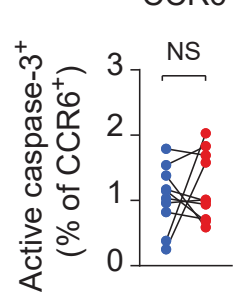

GC B

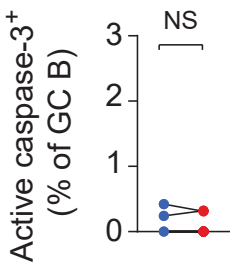

d
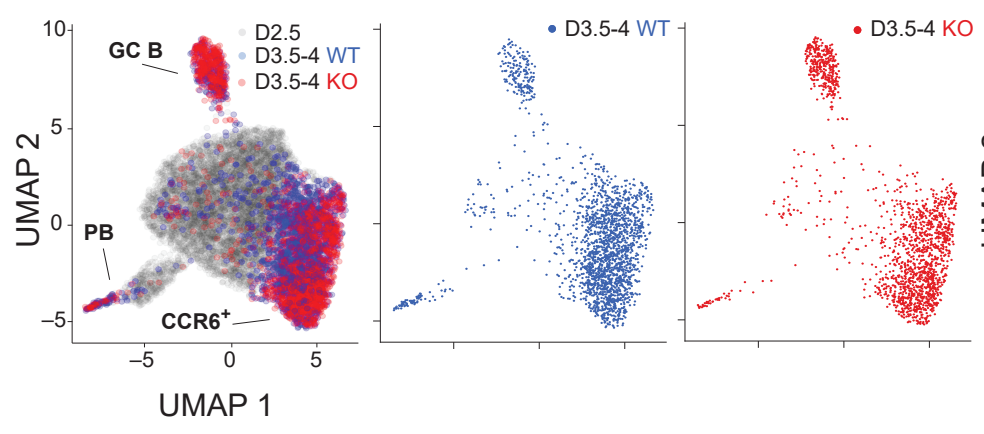

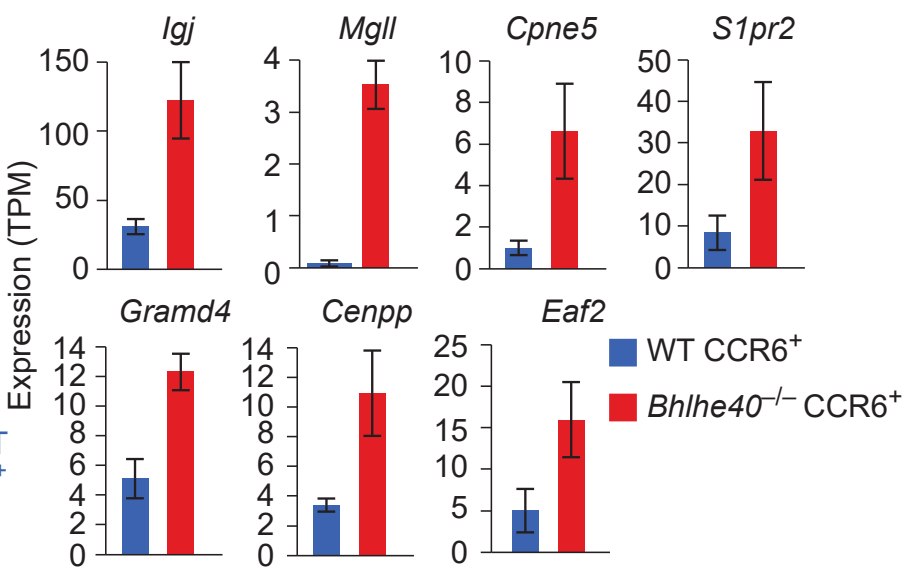

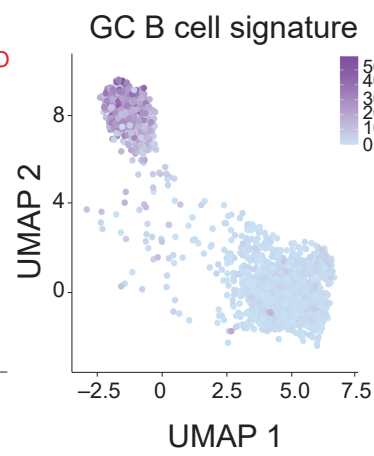

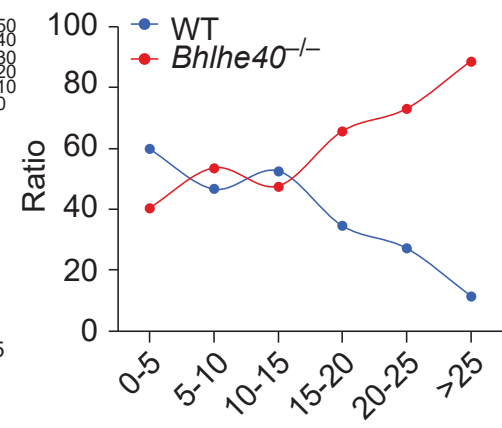

Expression of GC B cell signature GC B cell differentiation

Figure 3 
a

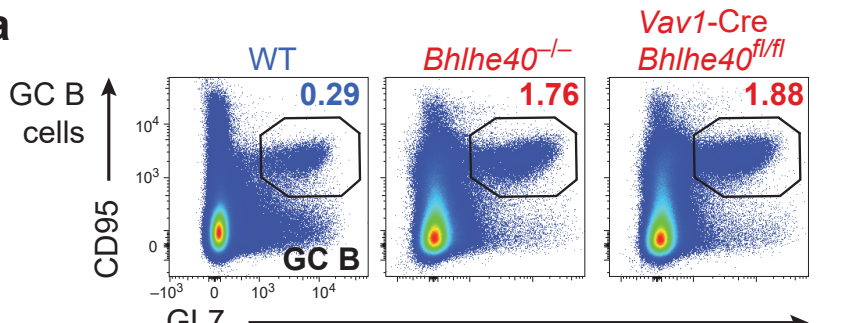
GL7
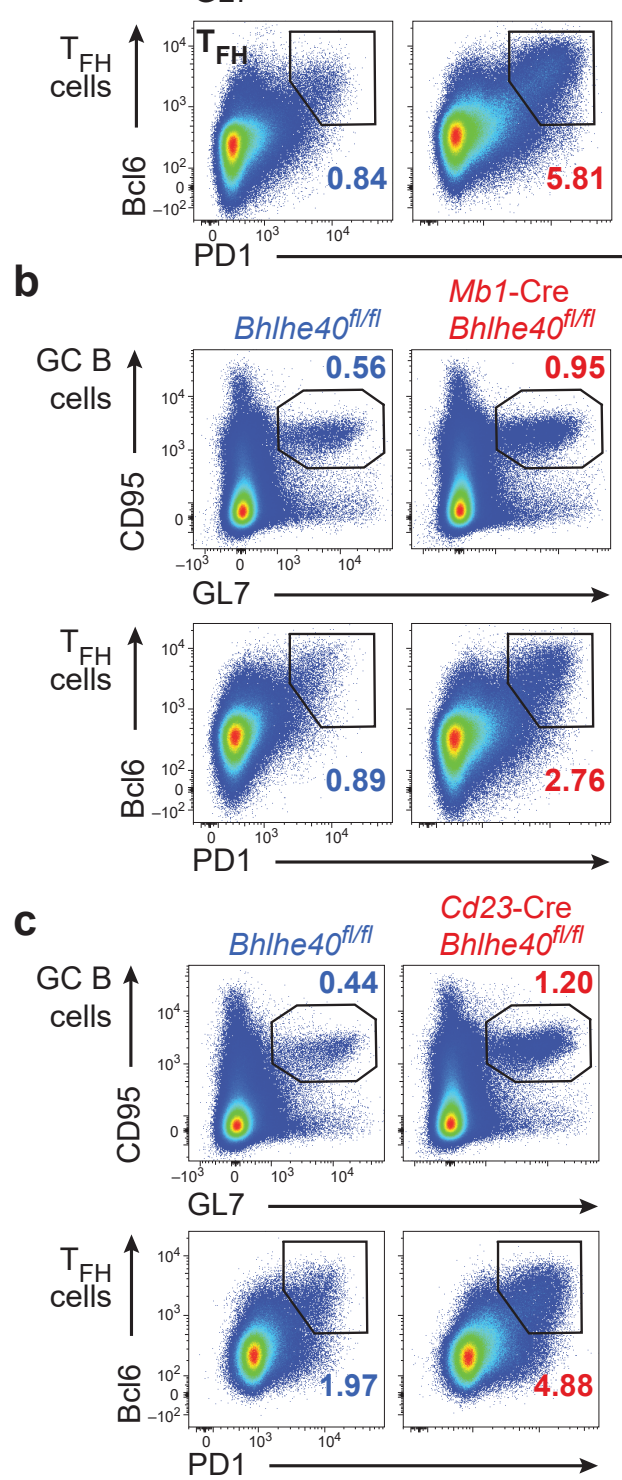
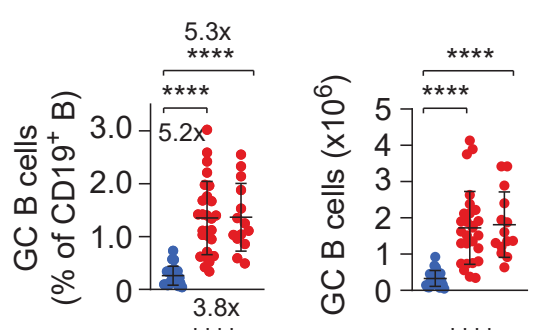

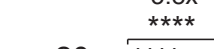

$\stackrel{F}{F}]^{\stackrel{* * * *}{\stackrel{*}{*}}}$

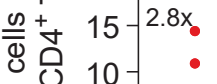

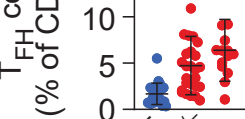

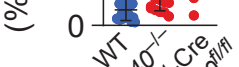

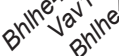
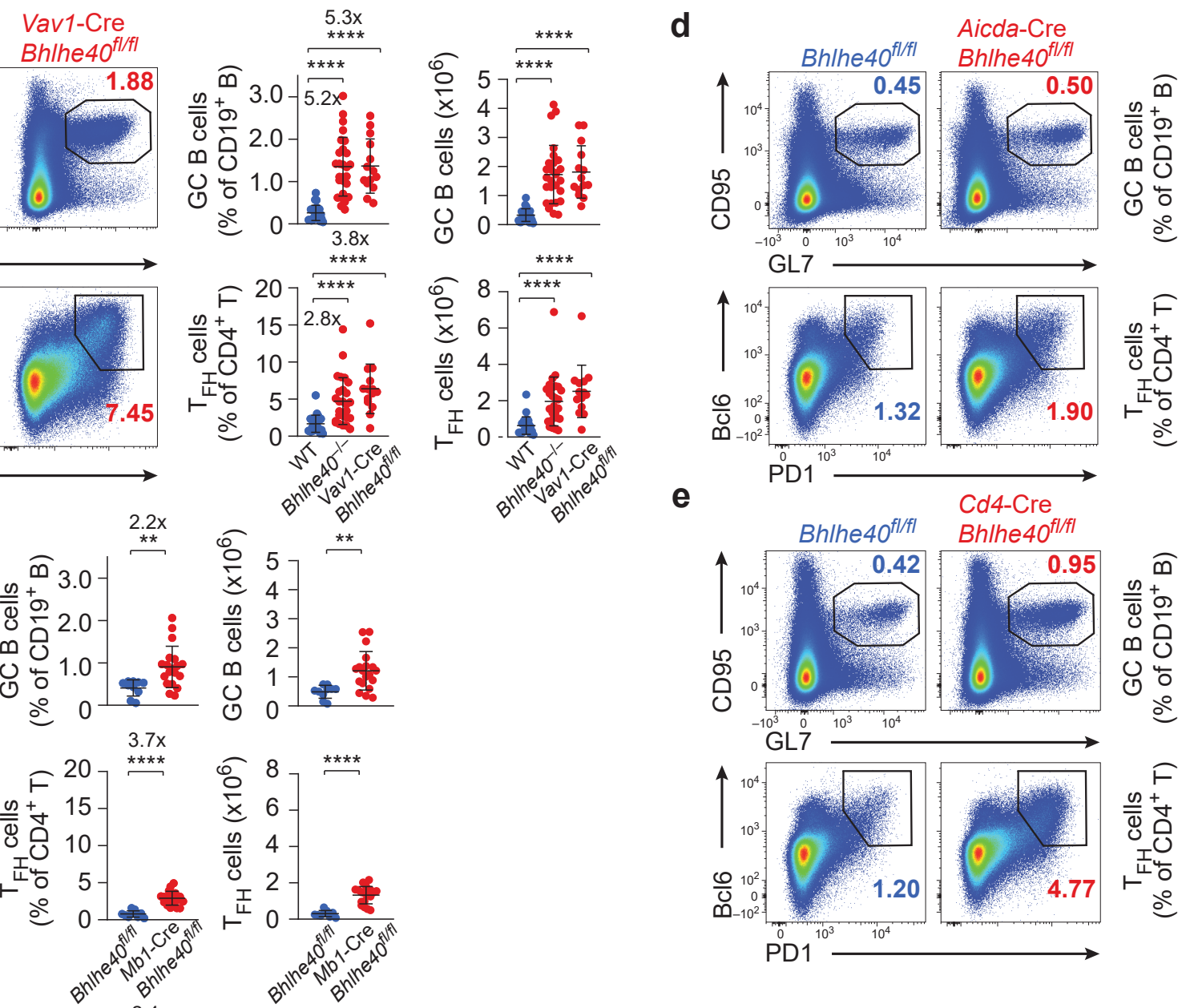

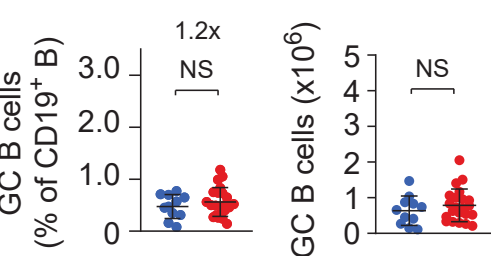
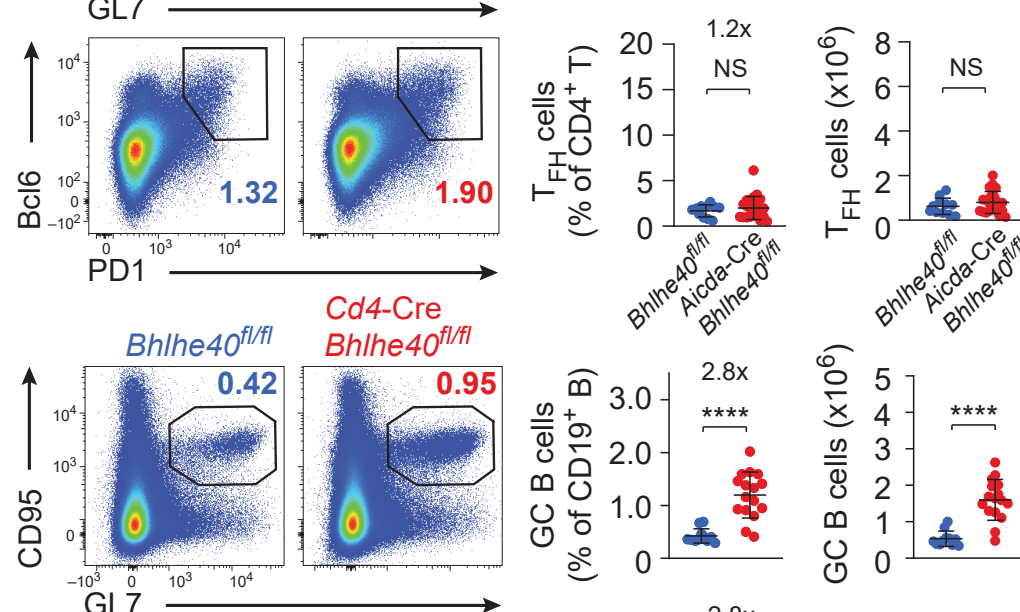

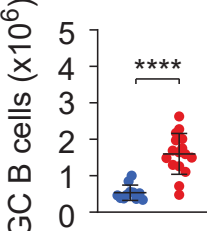
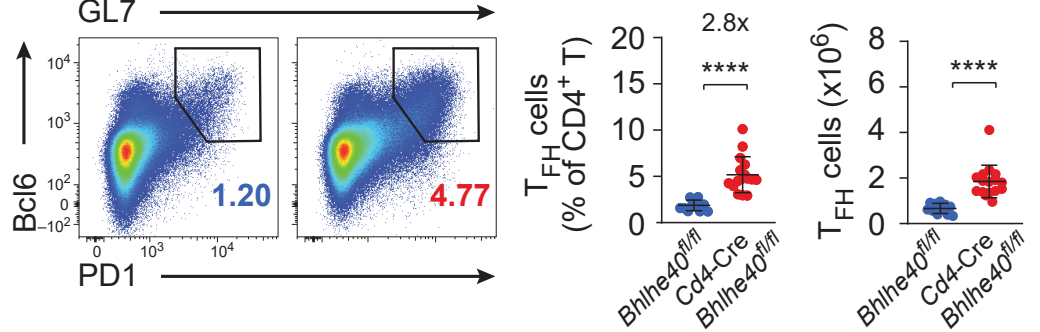
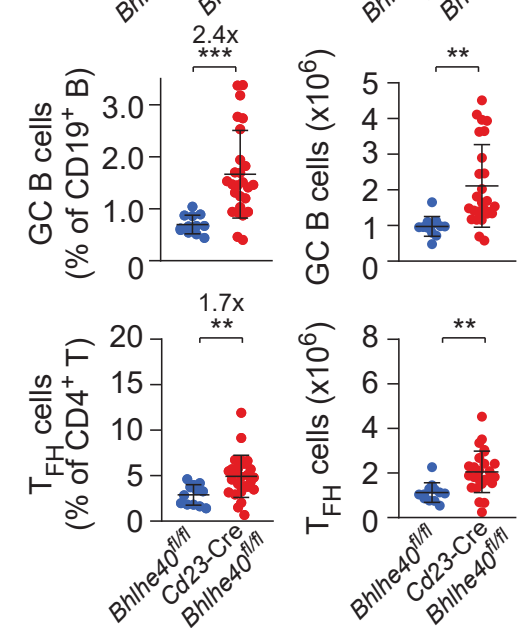

Figure 4 
a

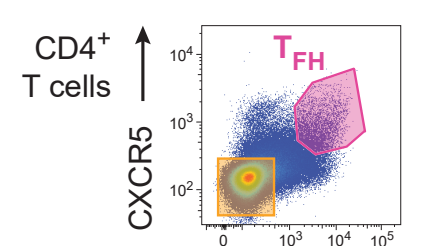

PD1

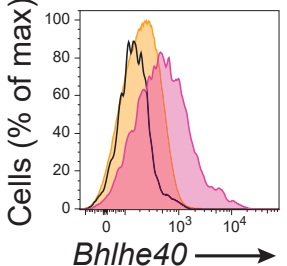

C

Mixed BM chimera

$\mathrm{Ag} 85 \mathrm{~b}_{280-294}$ peptide immunization, Day 11

$\mathrm{T}_{\mathrm{FH}}$ cells

PD $1^{-}$CXCR5 ${ }^{-}$cells

$\square \mathrm{T}_{\mathrm{FH}}$ cells, no probe
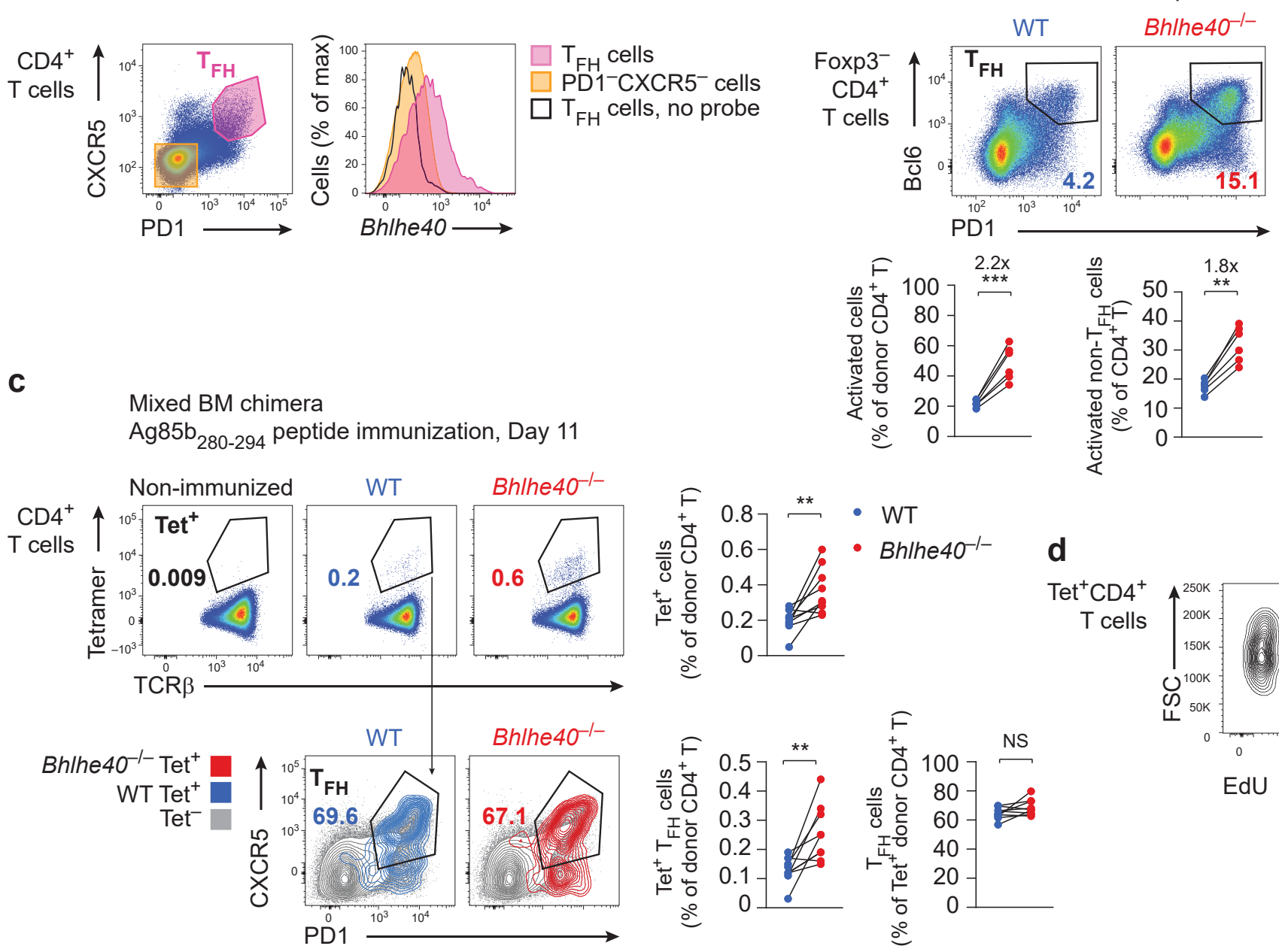

e

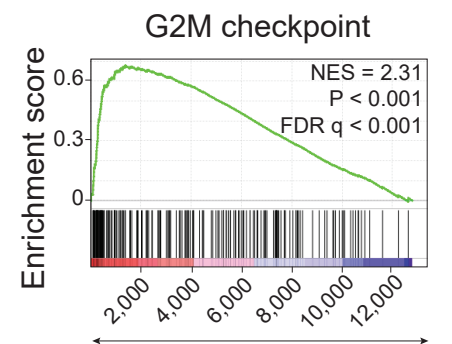

Up in Bh/he40 $0^{-/}$

Up in WT
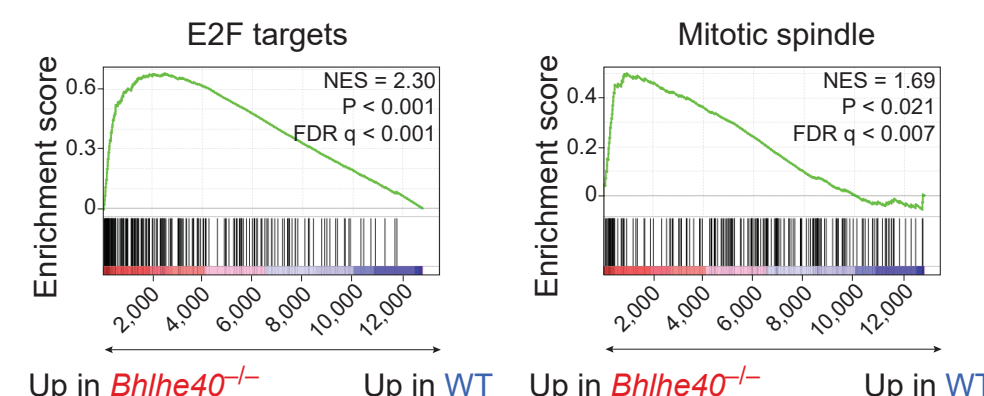

Figure 5
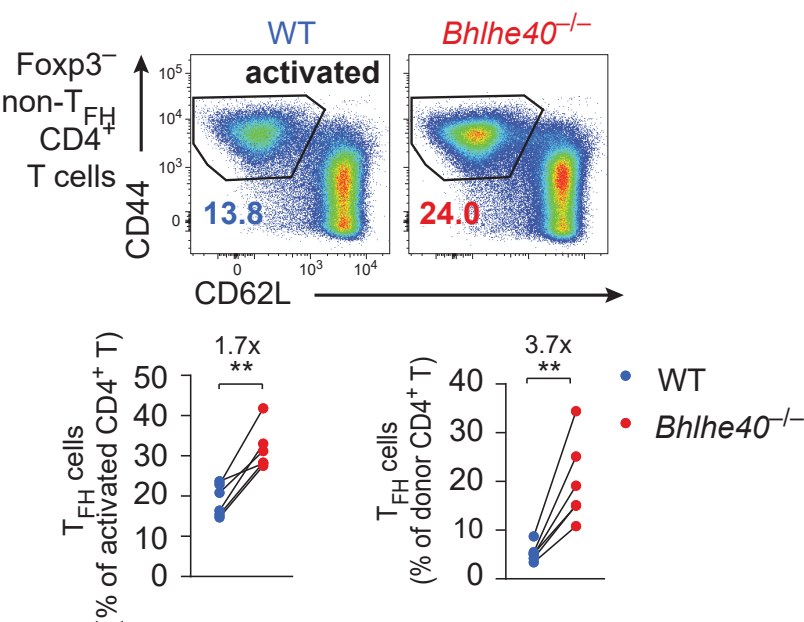

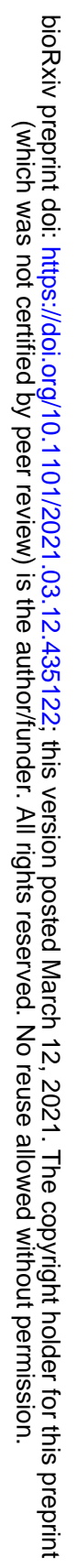

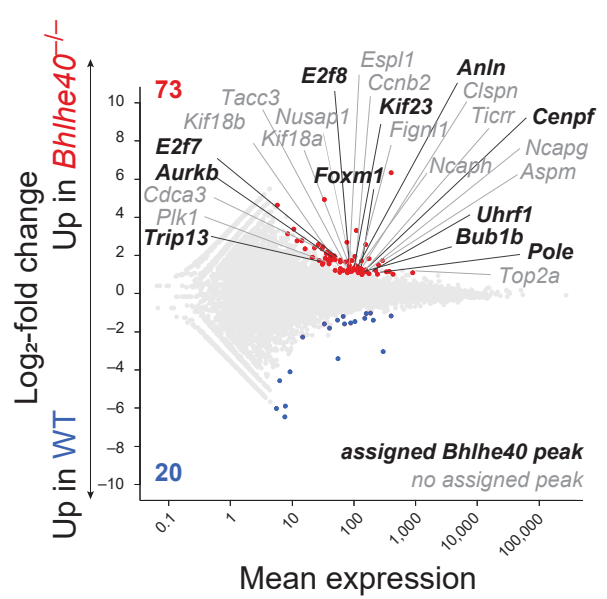


bioRxiv preprint doi: https://doi.org/10.1101/2021.03.12.435122; this version posted March 12, 2021. The copyright holder for this preprint (which was not certified by peer review) is the author/funder. All rights reserved. No reuse allowed without permission.

a

b

Bhlhe $40^{-/}, 15$ month

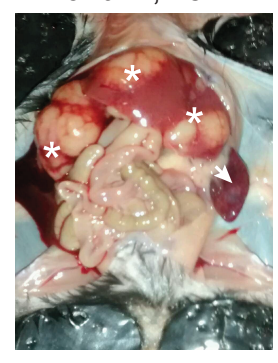

C

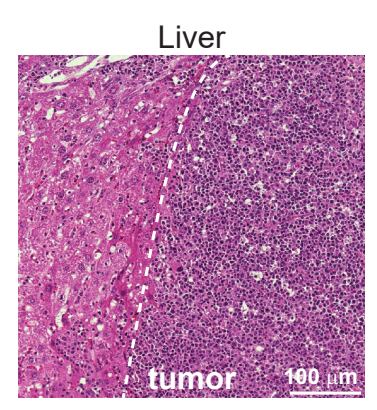

Bhlhe $40^{-1-}$ tumors

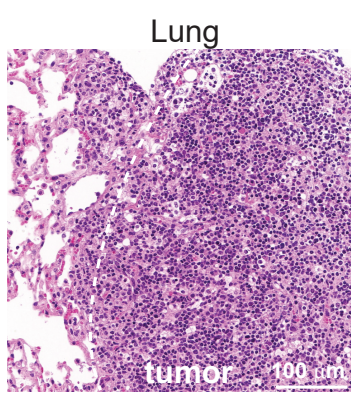

d

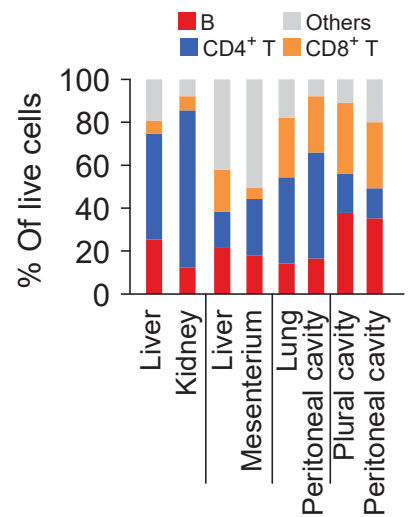

Mouse $\quad 1 \quad 2 \quad 3 \quad 4$

e

Bhlhe $40^{-1-}$ tumors f

g

$9 \quad B h l h e 40^{-1-}$ tumors

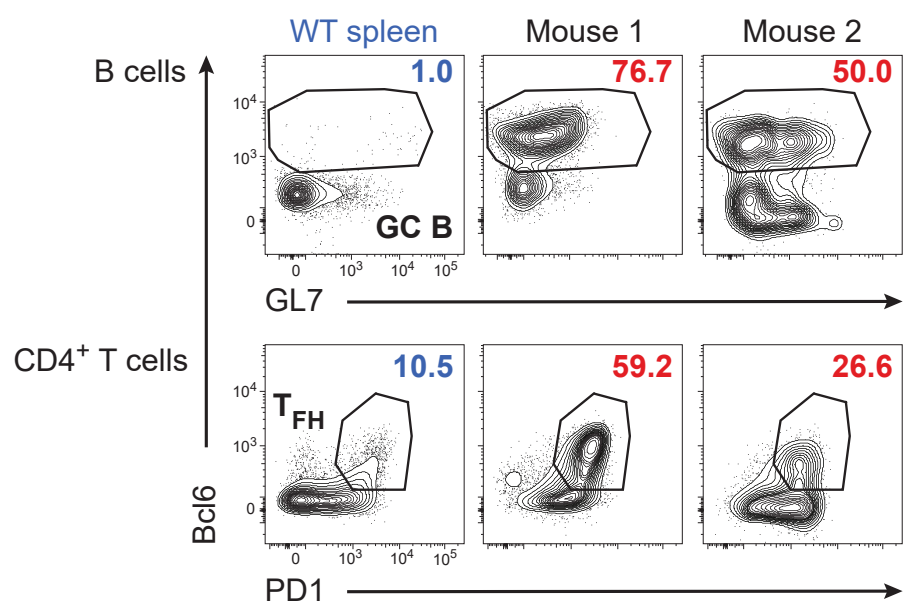

Bhlhe $40^{-1-}$ tumor (liver)
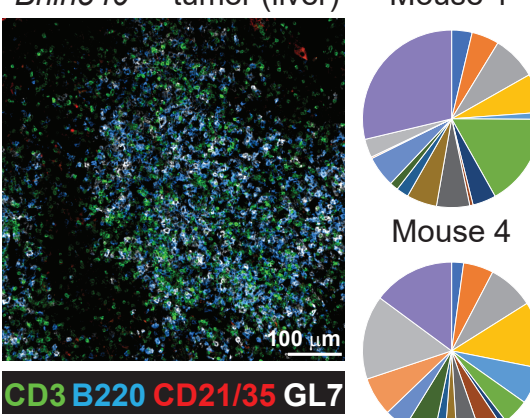

Mouse 4
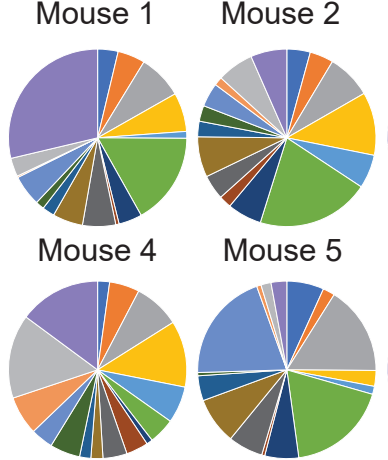

Mouse 5
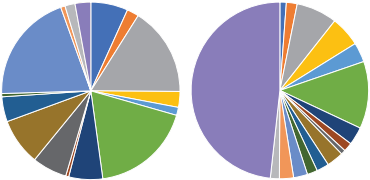

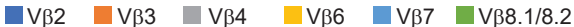

$\square \vee \beta 8.3 \square \vee \beta 9 \quad \square$ V $\beta 10 \quad \square \vee \beta 11 \quad \square \vee \beta 12$

a $\beta 13 \square \mathrm{V} \beta 14 \square \mathrm{V} \beta 17 \mathrm{a}=\mathrm{V} \beta 5.1 / 5.2 \quad$ Others h

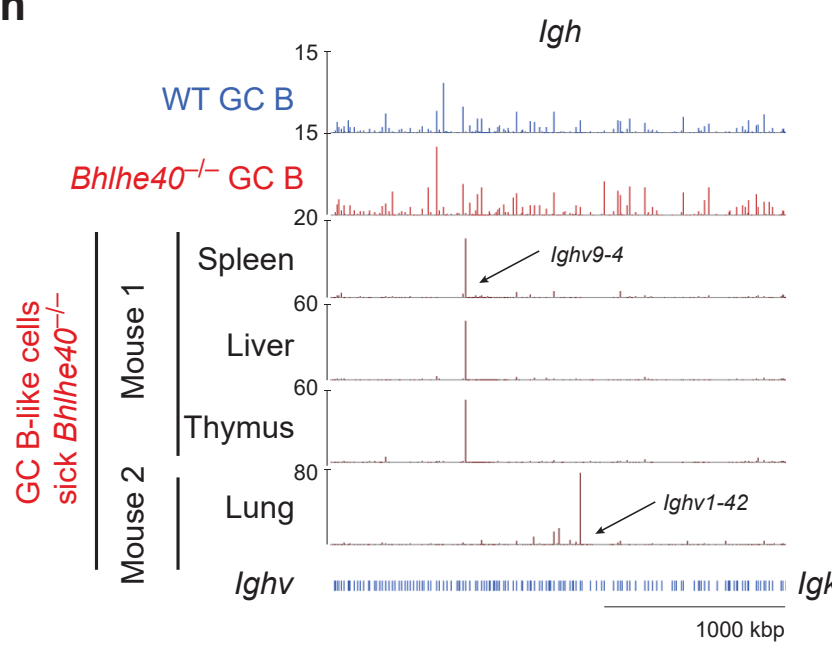

lgk

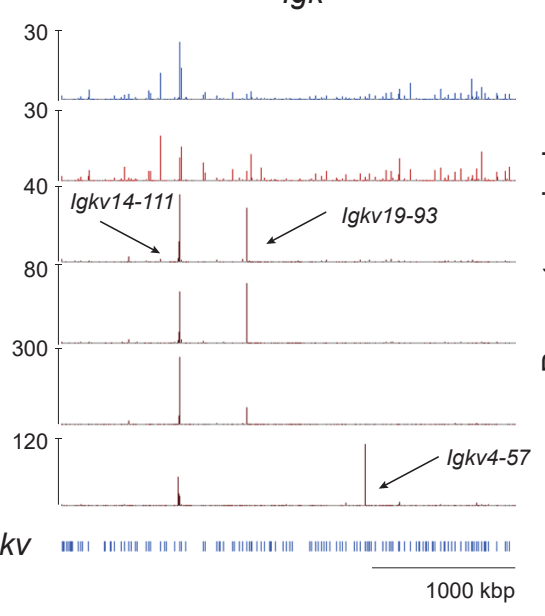

i Transfer of sorted tumor B and T cells

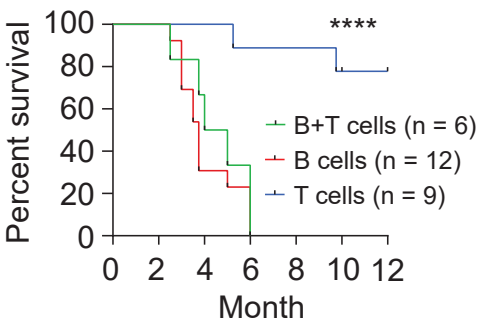

Figure 6 